\title{
Invariant conserved currents in generalized gravity
}

\author{
Yuri N. Obukhov* \\ Theoretical Physics Laboratory, Nuclear Safety Institute, \\ Russian Academy of Sciences, B.Tulskaya 52, 115191 Moscow, Russia \\ Felipe Portales-Oliva ${ }^{\dagger}$ \\ Departamento de Física, Universidad de Concepción, Casilla 160-C, Concepción, Chile \\ Dirk Puetzfeld ${ }^{\ddagger}$ \\ ZARM, University of Bremen, Am Fallturm, 28359 Bremen, Germany \\ Guillermo F. Rubilar ${ }^{\S}$ \\ Departamento de Física, Universidad de Concepción, Casilla 160-C, Concepción, Chile
}

(Dated: June 18, 2018)

\begin{abstract}
We study conservation laws for gravity theories invariant under general coordinate transformations. The class of models under consideration includes Einstein's general relativity theory as a special case as well as its generalizations to non-Riemannian spacetime geometry and nonminimal coupling. We demonstrate that an arbitrary vector field on the spacetime manifold generates a current density that is conserved under certain conditions, and find the expression of the corresponding superpotential. For a family of models including nonminimal coupling between geometry and matter, we discuss in detail the differential conservation laws and the conserved quantities defined in terms of covariant multipole moments. We show that the equations of motion for the multipole moments of extended microstructured test bodies lead to conserved quantities that are closely related to the conserved currents derived in the field-theoretic framework.
\end{abstract}

PACS numbers: 04.50.-h; 04.20.Fy; 04.20.Cv

Keywords: Metric-affine gravity, Conservation laws, Noether theorem, Variational methods.

\section{INTRODUCTION}

The correct understanding of the energy, momentum, and angular momentum in gravity theories is a prominent physical problem that has a long and complicated history. In order to solve this problem, numerous formalisms were developed to derive what are generally known as the conservation laws. There are two classes of conservation laws in any gravity theory. One class of conservation laws is formulated solely in terms of the dynamical variables that describe the gravitational field itself. These variables characterize the geometry of spacetime without involving additional structures of physical (nongeometrical) nature. The resulting conserved charges do not have tensor transformation properties under general coordinate transformations.

There is, however, another class of conservation laws that lead to conserved charges and that turn out to be true scalars. In deriving such conservation laws, one usually has to deal with, besides the gravitational field variables, additional physical structures such as vector fields. As it is well known, vector fields generate diffeomorphisms on a spacetime manifold. What is more

\footnotetext{
*obukhov@ibrae.ac.ru

† fportales@udec.cl

¥ dirk.puetzfeld@zarm.uni-bremen.de; http://puetzfeld.org

$\S$ grubilar@udec.cl
}

important, one can associate with a vector field $\zeta^{i}$ a current that is conserved under some conditions. In Einstein's general relativity theory this can be illustrated as follows.

Given a covariantly conserved symmetric energymomentum tensor, $\widetilde{\nabla}_{i} T_{k}{ }^{i}=0$, we find (contracting with $\left.\zeta^{k}\right)$ a relation: $\widetilde{\nabla}_{i} J^{i}=\frac{1}{2} T^{i j} \mathcal{L}_{\zeta} g_{i j}$. Here we defined the current $J^{i}:=\zeta^{k} T_{k}{ }^{i}$; furthermore, $\widetilde{\nabla}_{j}$ is the Riemannian covariant derivative and $\mathcal{L}_{\zeta}$ is the Lie derivative along the vector field $\zeta^{k}$. If $\zeta^{k}$ is a Killing vector, the current is conserved $\widetilde{\nabla}_{i} J^{i}=0$. This fact establishes a remarkable relation between the symmetries of the spacetime and the conserved currents generated by these symmetries. Physically, the vector field is usually related to the reference frame motion of an observer.

In this paper we extend this observation to a general framework of gravity theories that encompass possible non-Riemannian geometries and nonminimal couplings of matter to the gravitational field. Theories of this kind attracted considerable attention in the literature.

The history of the construction of conserved quantities in relativistic gravity is rich and long. The earliest relevant construction is the Komar charge and its close relatives [1-3]. Essential contributions to this approach include [4-19]. Most of these works were confined to the purely Riemannian geometrical framework of Einstein's general relativity. Extensions to more general geometries were studied in [20-26] and more recently by us [27-29] and [30-32]. 
The structure of the paper is as follows. In the next Sec. II we give an overview of the basic geometrical notions and operations which underlie our study. Sec. III presents the essentials of generalized gravity theory. We give a detailed derivation of the currents associated with the vector fields (diffeomorphisms) on the spacetime manifold, and specify the conditions under which these currents are conserved. In particular, we demonstrate the importance of generalized Killing vector fields, the properties of which we discuss in Sec. IV. In Sec. V we use our findings to obtain the generalized conserved current in metric-affine gravity (MAG) with a possible nonminimal coupling. Finally, we also show that the equations of motion for extended microstructured test bodies also admit a conserved quantity that is closely related (and has a similar structure) to the conserved currents derived in the field-theoretic framework. We conclude our paper in Sec. VI with a discussion of the results obtained and with an outlook of their possible applications.

Our notations and conventions are summarized at the end of the paper, in Appendix A.

\section{PRELIMINARIES: SPACETIME GEOMETRY}

In the metric-affine theory of gravity, the gravitational physics is described by the dynamics of the geometrical structure of spacetime. The latter is encoded in two fields: the metric tensor $g_{i j}$ and an independent linear connection $\Gamma_{k i}{ }^{j}$. The latter is not necessarily symmetric and/or compatible with the metric. From the geometrical point of view, the metric introduces lengths and angles of vectors, and thereby determines the distances (intervals) between points on the spacetime manifold. The connection introduces the notion of parallel transport and defines the covariant differentiation $\nabla_{k}$ of tensor fields.

Locally, a spacetime diffeomorphism can be described as a small translation in the spacetime manifold, which technically is represented by the variation of the spacetime coordinates

$$
\delta x^{i}=\epsilon \varepsilon^{i}(x) .
$$

Here $\epsilon$ is an infinitesimal constant parameter and $\varepsilon^{i}(x)$ is an arbitrary, but finite, vector field. Under the action of diffeomorphisms, the geometrical variables transform as

$$
\begin{aligned}
\delta g_{i j}= & -\epsilon\left(\partial_{i} \varepsilon^{k}\right) g_{k j}-\epsilon\left(\partial_{j} \varepsilon^{k}\right) g_{i k}, \\
\delta \Gamma_{k i}{ }^{j}= & -\epsilon\left(\partial_{k} \varepsilon^{l}\right) \Gamma_{l i}{ }^{j}-\epsilon\left(\partial_{i} \varepsilon^{l}\right) \Gamma_{k l}{ }^{j} \\
& +\epsilon\left(\partial_{l} \varepsilon^{j}\right) \Gamma_{k i}{ }^{l}-\epsilon \partial_{k i}^{2} \varepsilon^{j} .
\end{aligned}
$$

In general, the geometry of a metric-affine manifold is exhaustively characterized by three tensors: the curvature, the torsion and the nonmetricity. They are defined [33] as follows:

$$
\begin{aligned}
R_{k l i}{ }^{j} & :=\partial_{k} \Gamma_{l i}{ }^{j}-\partial_{l} \Gamma_{k i}{ }^{j}+\Gamma_{k n}{ }^{j} \Gamma_{l i}{ }^{n}-\Gamma_{l n}{ }^{j} \Gamma_{k i}{ }^{n}, \\
T_{k l}{ }^{i} & :=\Gamma_{k l}{ }^{i}-\Gamma_{l k}{ }^{i} \\
Q_{k i j} & :=-\nabla_{k} g_{i j}=-\partial_{k} g_{i j}+\Gamma_{k i}{ }^{l} g_{l j}+\Gamma_{k j}{ }^{l} g_{i l} .
\end{aligned}
$$

The curvature and the torsion tensors determine the commutator of the covariant derivatives. For a tensor $A^{c_{1} \ldots c_{k}} d_{1} \ldots d_{l}$ of arbitrary rank and index structure:

$$
\begin{aligned}
& \left(\nabla_{a} \nabla_{b}-\nabla_{b} \nabla_{a}\right) A^{c_{1} \ldots c_{k}} d_{1} \ldots d_{l}=-T_{a b}{ }^{e} \nabla_{e} A^{c_{1} \ldots c_{k}} d_{1} \ldots d_{l} \\
& +\sum_{i=1}^{k} R_{a b e^{c_{i}} A^{c_{1} \ldots e \ldots c_{k}} d_{1} \ldots d_{l}} \\
& -\sum_{j=1}^{l} R_{a b d_{j}}{ }^{e} A^{c_{1} \ldots c_{k}} d_{1} \ldots e \ldots d_{l} .
\end{aligned}
$$

The Ricci tensor is introduced by $R_{i j}:=R_{k i j}{ }^{k}$, and the curvature scalar is $R:=g^{i j} R_{i j}$.

A general metric-affine spacetime $\left(R_{k l i}{ }^{j} \neq 0, T_{k l}{ }^{i} \neq 0\right.$, $\left.Q_{k i j} \neq 0\right)$ incorporates several other spacetimes as special cases. The Riemannian connection $\widetilde{\Gamma}_{k j}{ }^{i}$ is uniquely determined by the conditions of vanishing torsion and nonmetricity which yield explicitly

$$
\widetilde{\Gamma}_{k j}^{i}=\frac{1}{2} g^{i l}\left(\partial_{j} g_{k l}+\partial_{k} g_{l j}-\partial_{l} g_{k j}\right)
$$

Here and in the following, a tilde over a symbol denotes a Riemannian object (such as the curvature tensor) or a Riemannian operator (such as the covariant derivative) constructed from the Christoffel symbols (8). The deviation of the geometry from the Riemannian one is then conveniently described by the distortion tensor

$$
N_{k j}^{i}:=\widetilde{\Gamma}_{k j}^{i}-\Gamma_{k j}^{i}
$$

The definitions (5) and (6) allows to find the distortion tensor in terms of the torsion and nonmetricity. Explicitly,

$N_{k j}^{i}=-\frac{1}{2}\left(T_{k j}^{i}+T_{k j}^{i}+T^{i}{ }_{j k}\right)+\frac{1}{2}\left(Q_{k j}^{i}-Q_{k j}{ }^{i}-Q_{j k}{ }^{i}\right)$.

Conversely, one can use this to express the torsion and nonmetricity tensors in terms of the distortion,

$$
\begin{aligned}
T_{k j}{ }^{i} & =-2 N_{[k j]}{ }^{i}, \\
Q_{k i j} & =-2 N_{k(i}{ }^{l} g_{j) l} .
\end{aligned}
$$

Substituting (9) into (4), we find the relation between the non-Riemannian and the Riemannian curvature tensors,

$R_{a d c}{ }^{b}=\widetilde{R}_{a d c}{ }^{b}-\widetilde{\nabla}_{a} N_{d c}{ }^{b}+\widetilde{\nabla}_{d} N_{a c}{ }^{b}+N_{a n}{ }^{b} N_{d c}{ }^{n}-N_{d n}{ }^{b} N_{a c}{ }^{n}$

Applying the covariant derivative to (4)-(6) and antisymmetrizing, we derive the Bianchi identities [33]:

$$
\begin{aligned}
\nabla_{[n} R_{k l] i}{ }^{j} & =T_{[k l}^{m} R_{n] m i}{ }^{j} \\
\nabla_{[n} T_{k l]}{ }^{i} & =R_{[k l n]}^{i}+T_{[k l}^{m} T_{n] m}{ }^{i} \\
\nabla_{[n} Q_{k] i j} & =R_{n k(i j)} .
\end{aligned}
$$




\section{A. Tensors, densities, and covariant differential operators}

Along with tensors, an important role in physics is played by densities. A fundamental density $\sqrt{-g}$ is constructed from the determinant of the metric, $g=\operatorname{det} g_{i j}$. Under diffeomorphisms (1) it transforms as

$$
\delta \sqrt{-g}=-\epsilon\left(\partial_{i} \varepsilon^{i}\right) \sqrt{-g} .
$$

This is a direct consequence of (2). From any tensor $B_{i \ldots}{ }^{j \ldots}$ one can construct a density $\mathfrak{B}_{i \ldots}{ }^{j \ldots}=$ $\sqrt{-g} B_{i \ldots}{ }^{j \ldots}$. Although in this paper we will encounter only such objects, it is worthwhile to notice that not all densities are of this type, since they can have different weights. The fundamental density $\sqrt{-g}$ and all other densities discussed here have weight +1 . See the exhaustive presentation in the book of Synge and Schild [34].

There are two kinds of covariant differential operators on the spacetime manifold, depending on whether the connection is involved or not. The Lie derivative $\mathcal{L}_{\varepsilon}$ is defined along any arbitrary vector field $\varepsilon^{i}$ and it maps tensors (densities) into tensors (densities) of the same rank. Let us recall the explicit form of the Lie derivative of the metric and the distortion:

$$
\begin{aligned}
\mathcal{L}_{\varepsilon} g_{i j}= & \varepsilon^{k} \partial_{k} g_{i j}+\left(\partial_{i} \varepsilon^{k}\right) g_{k j}+\left(\partial_{j} \varepsilon^{k}\right) g_{i k}, \\
\mathcal{L}_{\varepsilon} N_{k j}{ }^{i}= & \varepsilon^{n} \partial_{n} N_{k j}{ }^{i}+\left(\partial_{k} \varepsilon^{n}\right) N_{n j}{ }^{i} \\
& +\left(\partial_{j} \varepsilon^{n}\right) N_{k n}{ }^{i}-\left(\partial_{n} \varepsilon^{i}\right) N_{k j}{ }^{n} .
\end{aligned}
$$

In contrast, a covariant derivative $\nabla_{k}$ raises the rank of tensors (densities) and it is determined by the linear connection $\Gamma_{k j}{ }^{i}$. Moreover, there are different covariant derivatives which arise for different connections that may coexist on the same manifold.

A mathematical fact is helpful in this respect: every third rank tensor $X_{k j}{ }^{i}$ defines a map of one connection into a different new connection

$$
\Gamma_{k j}{ }^{i} \longrightarrow \Gamma_{k j}{ }^{i}+X_{k j}{ }^{i} .
$$

There are important special cases of such a map. One example is obtained for $X_{k j}{ }^{i}=N_{k j}{ }^{i}$ : then the connection $\Gamma_{k j}{ }^{i}$ is mapped into the Riemannian Christoffel symbols, $\widetilde{\Gamma}_{k j}{ }^{i}=\Gamma_{k j}{ }^{i}+N_{k j}{ }^{i}$, in accordance with (9).

Another interesting case arises for $X_{k j}{ }^{i}=T_{j k}{ }^{i}$. The result of the mapping

$$
\bar{\Gamma}_{k j}{ }^{i}=\Gamma_{k j}{ }^{i}+T_{j k}{ }^{i}=\Gamma_{k j}{ }^{i}+\Gamma_{j k}{ }^{i}-\Gamma_{k j}{ }^{i}=\Gamma_{j k}{ }^{i}
$$

is then called a transposed connection, or associated connection, see [35, 36].

The importance of the transposed connection is manifest in the following observation. Although the Lie derivative is a covariant operator - this is not apparent since it is based on partial derivatives - one can make everything explicitly covariant by noticing that it is possible to recast (18) and (19) into equivalent forms

$$
\begin{aligned}
\mathcal{L}_{\varepsilon} g_{i j}= & \varepsilon^{k} \nabla_{k} g_{i j}+\left(\bar{\nabla}_{i} \varepsilon^{k}\right) g_{k j}+\left(\bar{\nabla}_{j} \varepsilon^{k}\right) g_{i k}, \\
\mathcal{L}_{\varepsilon} N_{k j}{ }^{i}= & \varepsilon^{n} \nabla_{n} N_{k j}{ }^{i}+\left(\bar{\nabla}_{k} \varepsilon^{n}\right) N_{n j}{ }^{i} \\
& +\left(\bar{\nabla}_{j} \varepsilon^{n}\right) N_{k n}{ }^{i}-\left(\bar{\nabla}_{n} \varepsilon^{i}\right) N_{k j}{ }^{n} .
\end{aligned}
$$

By the same token we can "covariantize" the Lie derivatives for all other tensors of any structure and of arbitrary rank.

A more nontrivial (and less known) fact is that we can define the Lie derivatives also for objects which are not tensors. In particular, the Lie derivative of the connection then reads [35]:

$$
\begin{aligned}
\mathcal{L}_{\varepsilon} \Gamma_{k j}{ }^{i}= & \varepsilon^{l} \partial_{l} \Gamma_{k j}{ }^{i}+\left(\partial_{k} \varepsilon^{l}\right) \Gamma_{l j}^{i}+\left(\partial_{j} \varepsilon^{l}\right) \Gamma_{k l}^{i} \\
& -\left(\partial_{l} \varepsilon^{i}\right) \Gamma_{k j}{ }^{l}+\partial_{k j}^{2} \varepsilon^{i} \\
= & \nabla_{k} \bar{\nabla}_{j} \varepsilon^{i}-R_{k l j}{ }^{i} \varepsilon^{l} .
\end{aligned}
$$

This quantity measures the noncommutativity of the Lie derivative with the covariant derivative

$$
\begin{aligned}
& \left(\mathcal{L}_{\varepsilon} \nabla_{k}-\nabla_{k} \mathcal{L}_{\varepsilon}\right) A^{c_{1} \ldots c_{k}} d_{1} \ldots d_{l} \\
& =\sum_{i=1}^{k}\left(\mathcal{L}_{\varepsilon} \Gamma_{k b}{ }^{c_{i}}\right) A^{c_{1} \ldots b \ldots c_{k}} d_{1} \ldots d_{l} \\
& -\sum_{j-1}^{l}\left(\mathcal{L}_{\varepsilon} \Gamma_{k d_{j}}{ }^{b}\right) A^{c_{1} \ldots c_{k}} d_{1} \ldots b \ldots d_{l} .
\end{aligned}
$$

The connection $\Gamma_{k j}{ }^{i}$, the transposed connection $\bar{\Gamma}_{k j}{ }^{i}$, and the Riemannian connection $\widetilde{\Gamma}_{k j}{ }^{i}$ define the three respective covariant derivatives: $\nabla_{k}, \bar{\nabla}_{k}$, and $\widetilde{\nabla}_{k}$.

We will assume that these differential operators act on tensors. In addition, we will need the covariant operators that act on densities. For an arbitrary tensor density $\mathfrak{B}^{n}{ }_{i \ldots}{ }^{j \ldots}$ we introduce the covariant divergence

$\widehat{\nabla}_{n} \mathfrak{B}^{n}{ }_{i \ldots}{ }^{j \ldots}:=\partial_{n} \mathfrak{B}^{n}{ }_{i \ldots}{ }^{j \ldots}+\Gamma_{n l}{ }^{j} \mathfrak{B}^{n}{ }_{i \ldots}{ }^{l \ldots}-\Gamma_{n i}{ }^{l} \mathfrak{B}^{n}{ }_{l \ldots}{ }^{j \ldots}$,

which produces again a tensor density. We denote a similar differential operation constructed with the help of the Riemannian connection by

$\check{\nabla}_{n} \mathfrak{B}^{n}{ }_{i \ldots}{ }^{j \ldots}:=\partial_{n} \mathfrak{B}^{n}{ }_{i \ldots}{ }^{j \ldots}+\widetilde{\Gamma}_{n l}{ }^{j} \mathfrak{B}^{n}{ }_{i \ldots}{ }^{l \ldots}-\widetilde{\Gamma}_{n i}{ }^{l} \mathfrak{B}^{n}{ }_{l \ldots}{ }^{j \ldots}$,

When $\mathfrak{B}^{n}{ }_{i \ldots}{ }^{j \ldots}=\sqrt{-g} B^{n}{ }_{i \ldots}{ }^{j \ldots}$, we find

$$
\begin{aligned}
& \widehat{\nabla}_{n} \mathfrak{B}^{n}{ }_{i \ldots}{ }^{j \ldots}=\sqrt{-g}{ }^{*}{ }_{i} B^{n}{ }_{i \ldots .}{ }^{j \ldots}, \\
& \check{\nabla}_{n} \mathfrak{B}^{n}{ }_{i \ldots}{ }^{j \ldots}=\sqrt{-g} \widetilde{\nabla}_{n} B^{n}{ }_{i \ldots}{ }^{j \ldots},
\end{aligned}
$$

where we introduced a modified covariant derivative

$$
\stackrel{*}{\nabla}_{i}:=\nabla_{i}+N_{k i}{ }^{k} .
$$

A remark explaining our notation is in order. As one knows, a tensor is special case of a density with zero weight. So, strictly speaking, the introduction of the numerous superscript accents above may be viewed as something redundant. However, we find it more convenient to explicitly distinguish densities from tensors with the help of the "Fraktur" font for the former, and the "Roman" font for the latter. Accordingly we use different accented symbols for the derivatives. In particular, one should note that (31) acts on tensors, in contrast to (27) and (28) which act on densities. 


\section{B. Matter variables}

We will not specialize the discussion of matter to any particular physical field. It will be more convenient to describe matter by a generalized field $\psi^{A}$. The range of the indices $A, B, \ldots$ is not important in our study. However, we do need to know the behavior of the matter field under spacetime diffeomorphisms. We assume that under the transformation (1), these fields satisfy

$$
\delta \psi^{A}=-\epsilon\left(\partial_{i} \varepsilon^{j}\right)\left(\sigma^{A}{ }_{B}\right)_{j}{ }^{i} \psi^{B} .
$$

Here $\left(\sigma^{A}{ }_{B}\right)_{j}{ }^{i}$ are the generators of general coordinate transformations that satisfy the commutation relations

$$
\begin{aligned}
& \left(\sigma^{A}{ }_{C}\right)_{j}{ }^{i}\left(\sigma^{C}{ }_{B}\right)_{l}{ }^{k}-\left(\sigma^{A}{ }_{C}\right)_{l}{ }^{k}\left(\sigma^{C}{ }_{B}\right)_{j}{ }^{i} \\
& =\left(\sigma^{A}{ }_{B}\right)_{l}{ }^{i} \delta_{j}^{k}-\left(\sigma^{A}{ }_{B}\right)_{j}{ }^{k} \delta_{l}^{i} .
\end{aligned}
$$

We immediately recognize in (33) the Lie algebra of the general linear group $G L(4, R)$. This fact is closely related to the standard gauge-theoretic interpretation [37] of metric-affine gravity as the gauge theory of the general affine group $G A(4, R)$, which is a semidirect product of spacetime translation group times $G L(4, R)$.

The transformation properties (32) determine the form of the covariant and the Lie derivative of a matter field:

$$
\begin{aligned}
\nabla_{k} \psi^{A} & :=\partial_{k} \psi^{A}-\Gamma_{k i}{ }^{j}\left(\sigma^{A}{ }_{B}\right)_{j}{ }^{i} \psi^{B}, \\
\mathcal{L}_{\varepsilon} \psi^{A} & :=\varepsilon^{k} \partial_{k} \psi^{A}+\left(\partial_{i} \varepsilon^{j}\right)\left(\sigma^{A}{ }_{B}\right)_{j}{ }^{i} \psi^{B} \\
& =\varepsilon^{k} \nabla_{k} \psi^{A}+\left(\bar{\nabla}_{i} \varepsilon^{j}\right)\left(\sigma^{A}{ }_{B}\right)_{j}{ }^{i} \psi^{B} .
\end{aligned}
$$

The commutators of these differential operators read

$$
\begin{aligned}
\left(\nabla_{k} \nabla_{l}-\nabla_{l} \nabla_{k}\right) \psi^{A}= & -R_{k l j}{ }^{i}\left(\sigma^{A}{ }_{B}\right)_{i}{ }^{j} \psi^{B} \\
& -T_{k l}{ }^{i} \nabla_{i} \psi^{A}, \\
\left(\mathcal{L}_{\varepsilon} \nabla_{k}-\nabla_{k} \mathcal{L}_{\varepsilon}\right) \psi^{A}= & -\left(\mathcal{L}_{\varepsilon} \Gamma_{k j}{ }^{i}\right)\left(\sigma^{A}{ }_{B}\right)_{i}{ }^{j} \psi^{B} .
\end{aligned}
$$

\section{METRIC-AFFINE GRAVITY: FIELD EQUATIONS AND CURRENTS}

The explicit form of the dynamical equations of the gravitational field is irrelevant for the conservation laws that will form the basis for the derivation of the test body equations of motion. However, for completeness, we discuss here the field equations of a general metric-affine theory of gravity. The standard understanding of MAG is its interpretation as a gauge theory based on the general affine group $G A(4, R)$, which is a semidirect product of the general linear group $G L(4, R)$, and the group of local translations [37]. The corresponding gauge-theoretic formalism generalizes the approach of Sciama and Kibble $[38,39]$; for more details about gauge gravity theories, see [40-43]. Besides its many interesting properties, MAG offers the possibility of a unification of gravity with other physical interactions on the same gauge-theoretic principles, and contributes to the solution of the quantum gravity quest with encouraging attempts to construct a renormalizable theory of the quantized gravitational field [44-48].

In the standard formulation of MAG as a gauge theory [37], the gravitational gauge potentials are identified with the metric, coframe, and the linear connection. The corresponding gravitational field strengths are then the nonmetricity, the torsion, and the curvature, respectively.

It will be convenient to describe all the dynamical variables - including the gravitational (geometrical) and material fields - collectively by means of a multiplet, which we denote by $\Phi^{J}=\left(g_{i j}, \Gamma_{k i}{ }^{j}, \psi^{A}\right)$. The range of the multi-index $J$ is not important at present and will be specified when needed.

\section{A. Lagrange-Noether analysis}

The dynamics of the interacting gravitational and matter fields is determined by a general action

$$
I=\int d^{4} x \mathfrak{L}\left(\Phi^{J}, \partial_{i} \Phi^{J}\right)
$$

The Lagrangian density $\mathfrak{L}$ depends arbitrarily on its arguments. Let us investigate the variation of the action under transformations of the spacetime coordinates and the fields which, quite generally, read as follows:

$$
\begin{aligned}
x^{\prime i}(x) & =x^{i}+\delta x^{i}, \\
\Phi^{\prime J}\left(x^{\prime}\right) & =\Phi^{J}(x)+\delta \Phi^{J}(x) .
\end{aligned}
$$

Then, using the substantial variation defined by $\delta_{(s)} \Phi^{J}:=\Phi^{\prime J}(x)-\Phi^{J}(x)=\delta \Phi^{J}-\delta x^{k} \partial_{k} \Phi^{J}$, we derive in a standard way the total variation of the action

$$
\delta I=\int d^{4} x\left[\delta_{(s)} \mathfrak{L}+\partial_{i}\left(\mathfrak{L} \delta x^{i}\right)\right] .
$$

Assuming the invariance of the action under (40) and (41), we find the so-called Lie differential equation

$$
\delta_{(s)} \mathfrak{L}+\partial_{i}\left(\mathfrak{L} \delta x^{i}\right)=0 .
$$

With the help of the chain rule we can write

$$
\delta_{(s)} \mathfrak{L}=\frac{\partial \mathfrak{L}}{\partial \Phi^{J}} \delta_{(s)} \Phi^{J}+\frac{\partial \mathfrak{L}}{\partial\left(\partial_{i} \Phi^{J}\right)} \delta_{(s)} \partial_{i} \Phi^{J},
$$

and using the commutativity of the substantial variation with the partial derivative, $\delta_{(s)} \partial_{i}=\partial_{i} \delta_{(s)}$, we recast (43) into a balance equation

$$
\frac{\delta \mathfrak{L}}{\delta \Phi^{J}} \delta_{(s)} \Phi^{J}+\partial_{i}\left(\mathfrak{L} \delta x^{i}+\frac{\partial \mathfrak{L}}{\partial\left(\partial_{i} \Phi^{J}\right)} \delta_{(s)} \Phi^{J}\right)=0 .
$$

Here we denote the variational derivative

$$
\frac{\delta \mathfrak{L}}{\delta \Phi^{J}}:=\frac{\partial \mathfrak{L}}{\partial \Phi^{J}}-\partial_{i}\left(\frac{\partial \mathfrak{L}}{\partial\left(\partial_{i} \Phi^{J}\right)}\right),
$$

as usual. When the variational derivatives are put equal to zero, we obtain the system of classical field equations. 
The configurations of variables that satisfy $\delta \mathfrak{L} / \delta \Phi^{J}=0$ are called "on-shell".

The balance equation (45) is an identity, i.e. it is valid for all configurations of the gravitational and matter fields irrespectively of the fact that they are "on-shell" or "off-shell". Equation (45) gives rise to various identities and conservation laws which we derive in this paper.

We can apply (45) to different symmetries of the physical system under consideration. Of particular importance is the diffeomorphism (or general coordinate) invariance of the action (39). Substituting (1), (2), (3), and (32) into (45), we recast the latter (after dropping the overall infinitesimal constant $\epsilon$ ) into

$$
\Omega_{k} \varepsilon^{k}+\Omega_{k}{ }^{i} \partial_{i} \varepsilon^{k}+\frac{1}{2} \Omega_{k}{ }^{i j} \partial_{i j}^{2} \varepsilon^{k}+\frac{1}{3} \Omega_{k}{ }^{i j l} \partial_{i j l}^{3} \varepsilon^{k}=0 .
$$

The functions $\Omega_{k}{ }^{i_{1} \ldots i_{n}}$ (with $n=0,1,2,3$ ) are determined by the Lagrangian of the theory. Their explicit form is given in Appendix B.

In view of the arbitrariness of the vector field $\varepsilon^{k}$ and their derivatives, we obtain a set of Noether identities

$$
\Omega_{k}^{i_{1} \ldots i_{n}}=0
$$

for $n=0,1,2,3$.

\section{B. Generalized current}

Every vector field on the spacetime manifold induces a current which is conserved under certain conditions. We can derive it with the help of the balance equation (45) as follows. Let us consider a map of the manifold (diffeomorphism) induced by a vector field, as in Eq. (1). Locally, such a diffeomorphism acts on the gravitational and matter variables $\Phi^{J}=\left(g_{i j}, \Gamma_{k i}{ }^{j}, \psi^{A}\right)$ by means of the Lie derivatives so that

$$
\begin{aligned}
\delta_{(s)} g_{i j} & =-\epsilon \mathcal{L}_{\varepsilon} g_{i j}, \\
\delta_{(s)} \Gamma_{k j}{ }^{i} & =-\epsilon \mathcal{L}_{\varepsilon} \Gamma_{k j}{ }^{i}, \\
\delta_{(s)} \psi^{A} & =-\epsilon \mathcal{L}_{\varepsilon} \psi^{A} .
\end{aligned}
$$

Inserting this into (45) and dividing by the infinitesimal parameter $\epsilon$, we can define the current density

$$
\begin{aligned}
\mathfrak{J}^{i}:= & \frac{\partial \mathfrak{L}}{\partial \partial_{i} g_{k l}} \mathcal{L}_{\varepsilon} g_{k l}+\frac{\partial \mathfrak{L}}{\partial \partial_{i} \Gamma_{k n}{ }^{m}} \mathcal{L}_{\varepsilon} \Gamma_{k n}{ }^{m} \\
& +\frac{\partial \mathfrak{L}}{\partial \partial_{i} \psi^{A}} \mathcal{L}_{\varepsilon} \psi^{A}-\mathfrak{L} \varepsilon^{i} .
\end{aligned}
$$

and observe that it satisfies

$$
\partial_{i} \tilde{J}^{i}=-\frac{\delta \mathfrak{L}}{\delta g_{k l}} \mathcal{L}_{\varepsilon} g_{k l}-\frac{\delta \mathfrak{L}}{\delta \Gamma_{k n}{ }^{m}} \mathcal{L}_{\varepsilon} \Gamma_{k n}{ }^{m}-\frac{\delta \mathfrak{L}}{\delta \psi^{A}} \mathcal{L}_{\varepsilon} \psi^{A} .
$$

Using (18), (25), and (36), we can display the structure of the current as follows

$$
\mathfrak{J}^{i}=\varepsilon^{m} \mathfrak{J}_{m}{ }^{i}+\left(\bar{\nabla}_{n} \varepsilon^{m}\right) \mathfrak{J}_{m}{ }^{n i}+\left(\nabla_{k} \bar{\nabla}_{n} \varepsilon^{m}\right) \mathfrak{J}_{m}{ }^{k n i} .
$$

Here we introduced the densities

$$
\begin{aligned}
\mathfrak{J}_{m}{ }^{i}:= & \frac{\partial \mathfrak{L}}{\partial \partial_{i} \psi^{A}} \nabla_{m} \psi^{A}-\mathfrak{L} \delta_{m}^{i} \\
& -\frac{\partial \mathfrak{L}}{\partial \partial_{i} g_{k l}} Q_{m k l}-\frac{\partial \mathfrak{L}}{\partial \partial_{i} \Gamma_{k l}{ }^{n}} R_{k m l}{ }^{n}, \\
\mathfrak{J}_{m}{ }^{n i}:= & 2 \frac{\partial \mathfrak{L}}{\partial \partial_{i} g_{l n}} g_{l m}+\frac{\partial \mathfrak{L}}{\partial \partial_{i} \psi^{A}}\left(\sigma^{A}{ }_{B}\right)_{m}{ }^{n} \psi^{B}, \\
\mathfrak{J}_{m}{ }^{k n i}:= & \frac{\partial \mathfrak{L}}{\partial \partial_{i} \Gamma_{k n}{ }^{m}} .
\end{aligned}
$$

\section{Current and superpotential}

Expanding the Lie derivatives in (52), we can recast the current into a different equivalent form. Namely, a lengthy direct computation yields

$$
\begin{aligned}
\mathfrak{J}^{i}= & \Omega_{k}{ }^{i} \varepsilon^{k}+\Omega_{k}{ }^{n i} \partial_{n} \varepsilon^{k}+\Omega_{k}{ }^{m n i} \partial_{m n}^{2} \varepsilon^{k} \\
& -\varepsilon^{j}\left[2 \frac{\delta \mathfrak{L}}{\delta g_{i k}} g_{j k}+\frac{\delta \mathfrak{L}}{\delta \psi^{A}}\left(\sigma^{A}{ }_{B}\right)_{j}{ }^{i} \psi^{B}-\widehat{\nabla}_{k} \frac{\delta \mathfrak{L}}{\delta \Gamma_{k i}{ }^{j}}\right] \\
& -\left(\bar{\nabla}_{m} \varepsilon^{n}\right) \frac{\delta \mathfrak{L}}{\delta \Gamma_{i m}{ }^{n}}+\partial_{j} \mathfrak{K}^{i j} .
\end{aligned}
$$

Here we introduced a new density

$$
\begin{aligned}
\mathfrak{K}^{i j}:= & -\varepsilon^{k}\left[2 \frac{\partial \mathfrak{L}}{\partial \partial_{j} g_{i l}} g_{l k}+\frac{\partial \mathfrak{L}}{\partial \partial_{j} \psi^{A}}\left(\sigma^{A}{ }_{B}\right)_{k}{ }^{i} \psi^{B}\right. \\
& +\frac{\partial \mathfrak{L}}{\partial \Gamma_{j i}{ }^{k}}+\frac{\partial \mathfrak{L}}{\partial \partial_{j} \Gamma_{i n}{ }^{m}} \Gamma_{k n}{ }^{m}+\frac{\partial \mathfrak{L}}{\partial \partial_{j} \Gamma_{n i}{ }^{m}} \Gamma_{n k}{ }^{m} \\
& \left.-\frac{\partial \mathfrak{L}}{\partial \partial_{j} \Gamma_{n m^{k}}{ }^{k}} \Gamma_{n m}{ }^{i}-\partial_{l} \frac{\partial \mathfrak{L}}{\partial \partial_{(l} \Gamma_{j) i}{ }^{k}}\right] \\
& -\left(\partial_{n} \varepsilon^{k}\right)\left(\frac{\partial \mathfrak{L}}{\partial \partial_{j} \Gamma_{i n}{ }^{k}}+\frac{\partial \mathfrak{L}}{\partial \partial_{(n} \Gamma_{j) i}{ }^{k}}\right) .
\end{aligned}
$$

The first line on the right-hand side of (58) vanishes for the diffeomorphism invariant (generally covariant) theories in view of the Noether identities (48). As a result, we obtain "on-shell" (i.e., when $\delta \mathfrak{L} / \delta g_{i j}=0, \delta \mathfrak{L} / \delta \Gamma_{k j}{ }^{i}=0$, and $\left.\delta \mathfrak{L} / \delta \psi^{A}=0\right)$ :

$$
\mathfrak{J}^{i}=\partial_{j} \mathfrak{K}^{i j} .
$$

This means that on the classical field equations, the current $\mathfrak{J}^{i}$ is derived from the superpotential density $\mathfrak{K}^{i j}$.

\section{Explicitly covariant current}

It is not obvious that the complicated expressions (52), (58), and (59) are truly covariant objects under the action of diffeomorphisms. A direct demonstration is possible, but it is somewhat long to present it here. Instead, we will show the covariance by specifying the form of the Lagrangian. Intuitively it is clear (and can be rigorously proven) that the Lagrangian of a generally covariant theory should be a function of covariant objects. 
This means, in particular, that the derivatives of the basic variables $\Phi^{J}=\left(g_{i j}, \Gamma_{k i}{ }^{j}, \psi^{A}\right)$ should only appear in the form of explicitly covariant combinations. In simple terms,

$$
\mathfrak{L}\left(\Phi^{J}, \partial \Phi^{J}\right)=\mathfrak{L}\left(g_{i j}, Q_{k i j}, T_{i j}{ }^{k}, R_{i j k}^{l}, \psi^{A}, \nabla_{i} \psi^{A}\right) .
$$

We then immediately compute the partial derivatives

$$
\begin{aligned}
\frac{\partial \mathfrak{L}}{\partial \partial_{j} g_{i l}}= & -\frac{\partial \mathfrak{L}}{\partial Q_{j i l}} \\
\frac{\partial \mathfrak{L}}{\partial \partial_{j} \Gamma_{i n}{ }^{m}}= & 2 \frac{\partial \mathfrak{L}}{\partial R_{j i n}{ }^{m}} \\
\frac{\partial \mathfrak{L}}{\partial \partial_{j} \psi^{A}}= & \frac{\partial \mathfrak{L}}{\partial \nabla_{j} \psi^{A}} \\
\frac{\partial \mathfrak{L}}{\partial \Gamma_{k i}{ }^{j}}= & 2 \frac{\partial \mathfrak{L}}{\partial Q_{k i l}} g_{j l}+2 \frac{\partial \mathfrak{L}}{\partial T_{k i}{ }^{j}}-\frac{\partial \mathfrak{L}}{\partial \nabla_{k} \psi^{A}}\left(\sigma^{A}{ }_{B}\right)_{j}{ }^{i} \psi^{B} \\
& +2 \frac{\partial \mathfrak{L}}{\partial R_{k m n^{j}}{ }^{B}} \Gamma_{m n}{ }^{i}+2 \frac{\partial \mathfrak{L}}{\partial R_{m k i}{ }^{n}} \Gamma_{m j}{ }^{n}
\end{aligned}
$$

Substituting these expressions, we obtain the explicitly covariant current

$$
\begin{aligned}
\mathfrak{J}^{i}= & -\varepsilon^{j}\left[2 \frac{\delta \mathfrak{L}}{\delta g_{i k}} g_{j k}+\frac{\delta \mathfrak{L}}{\delta \psi^{A}}\left(\sigma^{A}{ }_{B}\right)_{j}{ }^{i} \psi^{B}-\widehat{\nabla}_{k} \frac{\delta \mathfrak{L}}{\delta \Gamma_{k i}{ }^{j}}\right] \\
& -\left(\bar{\nabla}_{m} \varepsilon^{n}\right) \frac{\delta \mathfrak{L}}{\delta \Gamma_{i m}{ }^{n}}+\check{\nabla}_{j} \mathfrak{K}^{i j} .
\end{aligned}
$$

and the explicitly covariant superpotential

$$
\mathfrak{K}^{i j}=2 \varepsilon^{k} \frac{\partial \mathfrak{L}}{\partial T_{i j}{ }^{k}}+2\left(\bar{\nabla}_{n} \varepsilon^{k}\right) \frac{\partial \mathfrak{L}}{\partial R_{i j n}{ }^{k}} .
$$

In our work [27], we studied the definition of conserved currents within models with local Lorentz invariance and vanishing nonmetricity. In those models, one can define different conserved currents depending on the choice of a so-called generalized Lie derivative. See [27, 29] for further details. On the other hand, the current (66) and the superpotential (67) are defined for any metricaffine model. Comparing both results for the restricted case of vanishing nonmetricity, one can verify after some straightforward algebra, that the superpotential (67) reduces to the components of the potential 2-form $H$ of [27], provided one uses the Yano choice for the generalized Lie derivative [49-51]. Therefore, the corresponding currents coincide when the classical field equations for the total system are satisfied. As a consequence, in the case of the Hilbert-Einstein Lagrangian, the conserved quantities reduce, in vacuum, to the well known Komar charges $[1,2]$.

\section{E. Conservation of the general current}

The current $\mathfrak{J}^{i}$ defined in (52), or equivalently in (66), is conserved when the right-hand side of the balance equation (53) vanishes,

$$
\partial_{i} \mathfrak{J}^{i}=0 .
$$

This is the case, for instance, for the "on-shell" field configuration when both the gravitational and matter variables satisfy the classical field equations $\delta \mathfrak{L} / \delta g_{i j}=0$, $\delta \mathfrak{L} / \delta \Gamma_{k j}{ }^{i}=0$, and $\delta \mathfrak{L} / \delta \psi^{A}=0$. Alternatively, the current is conserved, even "off-shell", when the Lie derivatives of all field variables vanish along a particular choice of the vector field $\varepsilon^{i}$. In these cases, when the vector field is not arbitrary, but some particular special case, we denote it by $\zeta^{i}$, so that it satisfies $\mathcal{L}_{\zeta} g_{i j}=0, \mathcal{L}_{\zeta} \Gamma_{k j}{ }^{i}=0$, and $\mathcal{L}_{\zeta} \psi^{A}=0$.

The last assumptions are, however, a bit too strong. A milder condition for the conservation of the current is the "on-shell" ansatz for the matter fields, $\delta \mathfrak{L} / \delta \psi^{A}=0$, combined with the vanishing of only the Lie derivatives for the gravitational fields along a vector field $\zeta^{i}: \mathcal{L}_{\zeta} g_{i j}=$ 0 and $\mathcal{L}_{\zeta} \Gamma_{k j}{ }^{i}=0$. We discuss the geometrical meaning of the latter conditions in Sec. IV.

Note also that (68) can be written in terms of the covariant derivatives $\widehat{\nabla}$ and $\check{\nabla}$ since, by the general definitions (27) and (28), we have

$$
\widehat{\nabla}_{i} \mathfrak{J}^{i}=\check{\nabla}_{i} \mathfrak{J}^{i}=\partial_{i} \mathfrak{J}^{i}
$$

\section{SYMMETRIES IN MAG: GENERALIZED KILLING VECTORS}

As is well known, symmetries of a Riemannian spacetime are generated by Killing vector fields. Each such field defines a so-called motion of the spacetime manifold, that is a diffeomorphism which preserves the metric $g_{i j}$.

Suppose $\zeta^{i}$ is a Killing vector field. By definition, it satisfies

$$
\widetilde{\nabla}_{i} \zeta_{j}+\widetilde{\nabla}_{j} \zeta_{i}=0 .
$$

By differentiation, we derive from this the second covariant derivative

$$
\widetilde{\nabla}_{i} \widetilde{\nabla}_{j} \zeta_{k}=\widetilde{R}_{j k i}{ }^{l} \zeta_{l} .
$$

We apply another covariant derivative and antisymmetrize:

$$
\widetilde{\nabla}_{[n} \widetilde{\nabla}_{i]} \widetilde{\nabla}_{j} \zeta_{k}=\widetilde{\nabla}_{[n}\left(\widetilde{R}_{|j k| i]}^{l} \zeta_{l}\right) .
$$

After some algebra, the last equation is recast into

$$
\begin{array}{r}
\zeta^{n} \widetilde{\nabla}_{n} \widetilde{R}_{i j k l}+\widetilde{R}_{n j k l} \widetilde{\nabla}_{i} \zeta^{n}+\widetilde{R}_{i n k l} \widetilde{\nabla}_{j} \zeta^{n} \\
+\widetilde{R}_{i j n l} \widetilde{\nabla}_{k} \zeta^{n}+\widetilde{R}_{i j k n} \widetilde{\nabla}_{l} \zeta^{n}=0 .
\end{array}
$$

Equations (70), (71), and (73) have a geometrical meaning:

$$
\begin{aligned}
\mathcal{L}_{\zeta} g_{i j} & =0, \\
\mathcal{L}_{\zeta} \widetilde{\Gamma}_{i j}{ }^{k} & =0 \\
\mathcal{L}_{\zeta} \widetilde{R}_{i j k l} & =0 .
\end{aligned}
$$


That is, the Lie derivatives along the Killing vector field $\zeta$ vanish for all Riemannian geometrical objects. Moreover, one can show that the same is true for all higher covariant derivatives of the Riemannian curvature tensor [49]:

$$
\mathcal{L}_{\zeta}\left(\widetilde{\nabla}_{n_{1}} \ldots \widetilde{\nabla}_{n_{N}} \widetilde{R}_{i j k l}\right)=0
$$

It is worthwhile to mention that in the Riemannian framework of Einstein's general relativity, one can define various symmetries generated by the vector fields (diffeomorphisms). For example, if $\zeta$ does not satisfy (74) but fulfills (75), such a vector field is not a Killing but a so-called called affine collineation. Alternatively, if both (74) and (75) are not true but $\zeta$ is characterized by the property (76), it is called a curvature collineation. Along with the standard Killing vector fields, these new fields contain important information about the symmetries of the spacetime, and it is possible to use them to define additional conservation laws. A comprehensive discussion of such symmetries (and related ones such as homothetic, conformal, projective, Ricci collineations etc) can be found in $[52,53]$, and different applications are studied in [54-56], for example.

Let us generalize the notion of a symmetry to the metric-affine spacetime. We take an ordinary Killing vector field $\zeta$ and postulate the vanishing of the Lie derivative

$$
\mathcal{L}_{\zeta} N_{k j}^{i}=0
$$

of the distortion tensor. Combining this with (10) and (75), we find an equivalent formulation

$$
\begin{aligned}
\mathcal{L}_{\zeta} g_{i j} & =0, \\
\mathcal{L}_{\zeta} \Gamma_{i j}{ }^{k} & =0 .
\end{aligned}
$$

We call a vector field that satisfies (79) and (80) a generalized Killing vector of the metric-affine spacetime. By definition, such a $\zeta$ generates a diffeomorphism of the spacetime manifold that is simultaneously an isometry (79) and an isoparallelism (80).

Since the Lie derivative along a Killing vector commutes with the covariant derivative, $\mathcal{L}_{\zeta} \widetilde{\nabla}_{i}=\widetilde{\nabla}_{i} \mathcal{L}_{\zeta}$, see (26), we conclude from (13) and (76) that the generalized Killing vector leaves the non-Riemannian curvature tensor invariant

$$
\mathcal{L}_{\zeta} R_{k l j}^{i}=0
$$

It is also straightforward to verify that

$$
\mathcal{L}_{\zeta}\left(\nabla_{n_{1}} \ldots \nabla_{n_{N}} R_{k l j}{ }^{i}\right)=0
$$

for any number of covariant derivatives of the curvature.

Finally, combining (11) and (12) with (74) and (78), we verify that

$$
\begin{gathered}
\mathcal{L}_{\zeta} T_{k j}{ }^{i}=0, \\
\mathcal{L}_{\zeta} Q_{k i j}=0 .
\end{gathered}
$$

Summarizing, the Lie derivative of all main geometrical objects vanishes along a generalized Killing vector field.

Later we will show that generalized Killing vectors have an important property: they induce conserved quantities on the metric-affine spacetime.

\section{MODIFIED MAG MODELS WITH A POSSIBLE NON-MINIMAL COUPLING}

\section{A. Gravitational field dynamics}

The general formalism which we developed in Sec. III will now be applied to specific models of the gravitational field coupled with matter. However, before we go into technical details, the following remark is in order. Strictly speaking, the metric-affine framework is applicable not only to generalized gravity theories which are based on the non-Riemannian spacetime geometries, but it is equally useful for the study of Einstein's GR and its modifications in a purely Riemannian geometrical context. Technically, this requires the introduction of additional variables which play the role of Lagrange multipliers, and this changes the physical meaning of the sources in the field equations. One should take note of this subtlety.

Here we use the formulation of MAG, in which gravity is described by the set of fundamental field variables which consists of the independent metric $g_{i j}$ and connection $\Gamma_{k i}{ }^{j}$. Such an approach was developed in [57-66], and this is alternative to the formalism which includes also a coframe field [37] as a gravitational field variable. It is instructive to compare the field equations in the different formulations of MAG, and in particular, it is necessary to clarify the role and place of the canonical energy-momentum tensor as a source of the gravitational field. Since one does not have the coframe (tetrad) among the fundamental variables, the corresponding field equation is absent. Here we demonstrate that one can always rearrange the field equations of MAG in such a way that the canonical energy-momentum tensor is recovered as one of the sources of the gravitational field.

For a large class of MAG models, the total Lagrangian of interacting gravitational and matter fields reads

$$
\mathfrak{L}=\mathfrak{V}+F \mathfrak{L}_{\text {mat }} .
$$

In general, the gravitational Lagrangian is constructed as a diffeomorphism covariant density function of the curvature, torsion, and nonmetricity,

$$
\mathfrak{V}=\mathfrak{V}\left(g_{i j}, R_{i j k}{ }^{l}, T_{k i}{ }^{j}, Q_{k i j}\right),
$$

whereas the matter Lagrangian depends on the matter fields $\psi^{A}$ and their covariant derivatives (34):

$$
\mathfrak{L}_{\text {mat }}=\mathfrak{L}_{\text {mat }}\left(g_{i j}, \psi^{A}, \nabla_{i} \psi^{A}\right) .
$$

In the current literature a considerable attention is paid to the study of so-called modified models in which 
one assumes a possibility that the gravitational field may interact with matter in a nonminimal way. Accordingly, we here also allow for nonminimal interaction of the matter to the gravitational field via the coupling function

$$
F=F\left(g_{i j}, R_{i j k}^{l}, T_{k i}{ }^{j}, Q_{k i j}\right) .
$$

When $F=1$, we recover the minimal coupling case. Let us write down the field equations of metric-affine gravity for that case. This can be done in several equivalent ways. The standard form is the set of the so-called "first" and "second" field equations. Using the covariant derivative for densities defined by (27), the field equations are given by

$$
\begin{aligned}
& \widehat{\nabla}_{n} \mathfrak{H}^{i n}{ }_{k}+\frac{1}{2} T_{m n}{ }^{i} \mathfrak{H}^{m n}{ }_{k}-\mathfrak{E}_{k}{ }^{i}=-\mathfrak{T}_{k}{ }^{i}, \\
& \widehat{\nabla}_{l} \mathfrak{H}^{k l i}{ }_{j}+\frac{1}{2} T_{m n}{ }^{k} \mathfrak{H}^{m n i}{ }_{j}-\mathfrak{E}^{k i}{ }_{j}=\mathfrak{S}^{i}{ }_{j}{ }^{k} .
\end{aligned}
$$

Here the generalized gravitational field momenta densities are introduced by

$$
\begin{aligned}
\mathfrak{H}^{k l i}{ }_{j} & :=-2 \frac{\partial \mathfrak{V}}{\partial R_{k l i}{ }^{j}}, \\
\mathfrak{H}^{k i}{ }_{j} & :=-\frac{\partial \mathfrak{V}}{\partial T_{k i}{ }^{j}} \\
\mathfrak{M}^{k i j} & :=-\frac{\partial \mathfrak{V}}{\partial Q_{k i j}}
\end{aligned}
$$

and the gravitational hypermomentum and the generalized energy-momentum densities are constructed as

$$
\begin{aligned}
\mathfrak{E}^{k i}{ }_{j}= & -\mathfrak{H}^{k i}{ }_{j}-\mathfrak{M}^{k i}{ }_{j}, \\
\mathfrak{E}_{k}{ }^{i}= & \delta_{k}^{i} \mathfrak{V}+\frac{1}{2} Q_{k l n} \mathfrak{M}^{i l n} \\
& +T_{k l}{ }^{n} \mathfrak{H}^{i l}{ }_{n}+R_{k l n}{ }^{m} \mathfrak{H}^{i l n}{ }_{m} .
\end{aligned}
$$

The sources of the gravitational field are the canonical energy-momentum tensor density and the canonical hypermomentum density of matter, respectively:

$$
\begin{aligned}
\mathfrak{T}_{k}{ }^{i} & :=\frac{\partial \mathfrak{L}_{\text {mat }}}{\partial \nabla_{i} \psi^{A}} \nabla_{k} \psi^{A}-\delta_{k}^{i} \mathfrak{L}_{\text {mat }}, \\
\mathfrak{S}^{i}{ }_{j}{ }^{k} & :=\frac{\partial \mathfrak{L}_{\text {mat }}}{\partial \Gamma_{k i}{ }^{j}}=-\frac{\partial \mathfrak{L}_{\text {mat }}}{\partial \nabla_{k} \psi^{A}}\left(\sigma^{A}{ }_{B}\right)_{j}{ }^{i} \psi^{B} .
\end{aligned}
$$

The usual spin density arises as the antisymmetric part of the hypermomentum,

$$
\tau_{i j}{ }^{k}:=\mathfrak{S}_{[i j]}{ }^{k},
$$

whereas the trace $\mathfrak{S}^{k}=\mathfrak{S}^{i}{ }_{i}{ }^{k}$ is the dilation current density. The symmetric traceless part describes the proper hypermomentum [37].

It is straightforward to verify that instead of the first field equation (89), one can use the so-called zeroth field equation which reads

$$
2 \frac{\delta \mathfrak{V}}{\delta g_{i j}}=\mathfrak{t}^{i j}
$$

On the right-hand side, the matter source is now represented by the metrical energy-momentum tensor which is defined by

$$
\mathfrak{t}_{i j}:=2 \frac{\partial \mathfrak{L}_{\mathrm{mat}}}{\partial g^{i j}}
$$

The system (89) and (90) is completely equivalent to the system (99) and (90), and it is a matter of convenience which one to solve.

\section{B. Conservation laws}

The dynamics of extended bodies in metric-affine spaces can be derived by integrating the conservation laws. The latter are obtained from the Noether identities. After some algebra, the identities (48) are recast into the following set of conservation laws

$$
\begin{aligned}
F \mathfrak{T}_{k}{ }^{i}= & F \mathfrak{t}_{k}{ }^{i}+\widehat{\nabla}_{n}\left(F \mathfrak{S}^{i}{ }_{k}{ }^{n}\right), \\
\widehat{\nabla}_{i}\left(F \mathfrak{T}_{k}{ }^{i}\right)= & F\left(\mathfrak{T}_{l}{ }^{i} T_{k i}{ }^{l}-\mathfrak{S}^{m}{ }_{n}{ }^{l} R_{k l m}{ }^{n}-\frac{1}{2} \mathfrak{t}^{i j} Q_{k i j}\right) \\
& -\mathfrak{L}_{\text {mat }} \nabla_{k} F .
\end{aligned}
$$

These relations hold "on-shell" when the matter variables $\psi^{A}$ satisfy the classical field equations, and are valid for general nonminimal coupling.

\section{Rewriting the conservation laws}

Using (31) and decomposing the connection into the Riemannian and non-Riemannian parts, c.f. Eq. (9), we can recast the conservation law (101) into an equivalent form:

$\check{\nabla}_{j}\left(F \mathfrak{S}_{k}^{i}{ }^{j}\right)=F\left(\mathfrak{T}_{k}^{i}-\mathfrak{t}_{k}^{i}+N_{n m}{ }^{i} \mathfrak{S}_{k}^{m}{ }^{n}-N_{n k}{ }^{m} \mathfrak{S}_{m}^{i}{ }^{n}\right)$.

In a similar way we can rewrite the conservation law (102). At first, with the help of (11) and (12) we notice that

$$
F\left(\mathfrak{T}_{l}^{i} T_{k i}{ }^{l}-\frac{1}{2} \mathfrak{t}^{i j} Q_{k i j}\right)=F\left(\mathfrak{t}_{l}{ }^{i}-\mathfrak{T}_{l}{ }^{i}\right) N_{k i}{ }^{l}+F \mathfrak{T}_{l}^{i} N_{i k}{ }^{l} .
$$

Then substituting here (103) and making use of (31), (9), and the curvature decomposition (13), after some algebra we recast (102) into

$$
\begin{aligned}
\check{\nabla}_{j}\left\{F\left(\mathfrak{T}_{k}{ }^{j}+\mathfrak{S}^{m}{ }_{n}{ }^{j} N_{k m}{ }^{n}\right)\right\} \\
=-F \mathfrak{S}^{m}{ }_{n}{ }^{i}\left(\widetilde{R}_{k i m}{ }^{n}-\widetilde{\nabla}_{k} N_{i m}{ }^{n}\right) \\
\quad-\mathfrak{L}_{\text {mat }} \widetilde{\nabla}_{k} F .
\end{aligned}
$$

For the minimal coupling case, such a conservation law was derived in $[27,67]$. The importance of this form of the energy-momentum conservation law lies in the clear 
separation of the Riemannian and non-Riemannian geometrical variables. As we see, the post-Riemannian geometry enters (105) only in the form of the distortion tensor $N_{k j}{ }^{i}$ which is coupled only to the hypermomentum current density $\mathfrak{S}^{m}{ }_{n}{ }^{i}$. This means that, in the minimal coupling case, ordinary matter - i.e. without microstructure, $\mathfrak{S}^{m}{ }_{n}{ }^{i}=0$ - does not couple to the non-Riemannian geometry. In contrast, in the nonminimal coupling case, the derivative of the coupling function $F$ on the righthand side of (105) may lead to a coupling between nonRiemannian structures and ordinary matter.

\section{Conserved current induced by a spacetime symmetry}

As we have shown, every vector field generates a conserved current. It is straightforward to show that for a Lagrangian density of the form $\mathfrak{L}=F \mathfrak{L}_{\text {mat }}$, as defined in (87) and (88), if we consider a generalized Killing vector field $\zeta^{k}$, the current (54) reads,

$$
\mathfrak{J}^{i}:=F\left[\zeta^{k} \mathfrak{T}_{k}^{i}-\left(\bar{\nabla}_{m} \zeta^{n}\right) \mathfrak{S}_{n}^{m}{ }_{n}^{i}\right] .
$$

Here we have used the definitions (96) and (97), and the conditions (79) and (80).

It is instructive to derive this result directly from the field equations. Namely, let us contract equation (103) with $\widetilde{\nabla}_{i} \zeta^{k}$ and contract equation (105) with $\zeta^{k}$, and then subtract the resulting expressions. Note that the contraction $\mathfrak{t}_{k}{ }^{i} \widetilde{\nabla}_{i} \zeta^{k}=0$ vanishes because the first factor is a symmetric tensor and the second one is skew-symmetric. Then after some algebra we find

$$
\check{\nabla}_{i} \mathfrak{J}^{i}=F \mathfrak{S}^{m}{ }_{n}^{i} \mathcal{L}_{\zeta} N_{i m}{ }^{n}-\mathfrak{L}_{\text {mat }} \mathcal{L}_{\zeta} F .
$$

The right-hand side of (107) depends linearly on the Lie derivatives along the Killing vector: $\mathcal{L}_{\zeta} F$ and $\mathcal{L}_{\zeta} N_{i m}{ }^{n}$, see (19) and (23).

When $\zeta^{k}$ is a generalized Killing vector, we have $\mathcal{L}_{\zeta} N_{i m}{ }^{n}=0$ in view of (78). Furthermore, recalling that $F=F\left(g_{i j}, R_{k l j}{ }^{i}, T_{k i}{ }^{j}, Q_{k i j}\right)$, we find

$$
\begin{aligned}
\mathcal{L}_{\zeta} F= & \frac{\partial F}{\partial g_{i j}} \mathcal{L}_{\zeta} g_{i j}+\frac{\partial F}{\partial R_{k l j}{ }^{i}} \mathcal{L}_{\zeta} R_{k l j}{ }^{i} \\
& +\frac{\partial F}{\partial T_{k j}{ }^{i}} \mathcal{L}_{\zeta} T_{k j}{ }^{i}+\frac{\partial F}{\partial Q_{k i j}} \mathcal{L}_{\zeta} Q_{k i j}=0
\end{aligned}
$$

by making use of (74), (81), (83), and (84).

As a result, the right-hand side of (107) vanishes for the generalized Killing vector field, and we conclude that the induced current (106) is conserved.

This generalizes the earlier results reported in [27, 28, 36]. In Sec. VF we will show that there is a conserved quantity constructed from the multipole moments which is a direct counterpart of the induced current (106). It is worthwhile to give an equivalent form of the latter:

$$
\mathfrak{J}^{i}=F\left[\zeta^{k}\left(\mathfrak{T}_{k}^{i}+\mathfrak{S}^{m}{ }_{n}^{i} N_{k m}{ }^{n}\right)-\left(\widetilde{\nabla}_{m} \zeta^{n}\right) \mathfrak{S}_{n}^{m}{ }_{n}^{i}\right]
$$

\section{E. Test body equations of motion}

The details of the multipole approximation scheme are given elsewhere; see Appendix $\mathrm{C}$ for the definitions of the integrated moments. Here we limit ourselves to the poledipole equations of motion. Following [68], we introduce the total orbital and the total spin angular moments

$$
L^{a b}:=2 p^{[a b]}, \quad S^{a b}:=-2 h^{[a b]},
$$

where $p^{a b}$ and $h^{a b}$ are generalized multipolar moments. The generalized total energy-momentum 4-vector and the generalized total angular momentum are given by

$$
\begin{aligned}
\mathcal{P}^{a} & :=F\left(p^{a}+N^{a}{ }_{c d} h^{c d}\right)+p^{b a} \widetilde{\nabla}_{b} F, \\
\mathcal{J}^{a b} & :=F\left(L^{a b}+S^{a b}\right) .
\end{aligned}
$$

Then the pole-dipole equations of motion take the form

$$
\begin{aligned}
\frac{D \mathcal{P}^{a}}{d s}= & \frac{1}{2} \widetilde{R}_{b c d}^{a} v^{b} \mathcal{J}^{c d}+F q^{c b d} \widetilde{\nabla}^{a} N_{d c b} \\
& -\xi \widetilde{\nabla}^{a} F-\xi^{b} \widetilde{\nabla}_{b} \widetilde{\nabla}^{a} F \\
\frac{D \mathcal{J}^{a b}}{d s}= & -2 v^{[a} \mathcal{P}^{b]}+2 F\left(q^{c d[a} N^{b]}{ }_{c d}+q^{c[a|d|} N_{d c}{ }^{b]}\right. \\
& \left.+q^{[a|c d|} N_{d}^{b]}{ }_{c}\right)-2 \xi^{[a} \widetilde{\nabla}^{b]} F .
\end{aligned}
$$

Here $v^{a}$ is the 4-vector of velocity of the test particle, $\frac{D}{d s}=v^{a} \widetilde{\nabla}_{a}$, with $s$ the proper time of the particle, whereas $q^{a b c}, \xi$ and $\xi^{a}$ are integrated moments relevant for the description. See App. C, as well as [68] for further details.

\section{F. Conserved quantity for extended test bodies}

The equations of motion for the multipole moments are derived from the conservation laws of the energymomentum and the hypermomentum currents $\mathfrak{T}_{k}{ }^{i}$ and $\mathfrak{S}^{m}{ }_{n}{ }^{i}$. In Sec. VD we have demonstrated that every generalized Killing vector induces a conserved current depending on these two quantities, see Eqs. (106) and (109). Quite remarkably, there is a direct counterpart of such an induced current built from the multipole moments.

Let $\zeta^{k}$ be a generalized Killing vector, and let us contract equation (113) with $\zeta_{a}$ and equation (114) with $\frac{1}{2} \widetilde{\nabla}_{a} \zeta_{b}$, and then take the sum. This yields

$$
\begin{aligned}
\frac{D}{d s}\left(\mathcal{P}^{a} \zeta_{a}+\frac{1}{2} \mathcal{J}^{a b} \widetilde{\nabla}_{a} \zeta_{b}\right) & =F q^{c b d} \mathcal{L}_{\zeta} N_{d c b} \\
& -\xi \mathcal{L}_{\zeta} F-\xi^{a} \widetilde{\nabla}_{a} \mathcal{L}_{\zeta} F
\end{aligned}
$$

On the right-hand side of (115) the Lie derivatives of the distortion tensor and of the coupling function vanish in view of (78) and (108).

Consequently, we conclude that for every generalized Killing vector field the quantity

$$
\mathcal{P}^{a} \zeta_{a}+\frac{1}{2} \mathcal{J}^{a b} \widetilde{\nabla}_{a} \zeta_{b}=\text { const. }
$$


is conserved along a trajectory of an extended body.

We thus observe a complete consistency between (106) and (116), as well as between (107) and (115).

The conserved quantities with the similar structure in (116) were derived previously for extended test bodies with mass and spin in Einstein's general relativity [6971] and in Einstein-Cartan gravity [36]. Their various applications were recently discussed in [72, 73].

\section{G. Relation between theories of fields and particles}

The similarity between the currents in a field-theoretic picture and the integrals of motion in particle mechanics is not occasional. Here we demonstrate that the current (106), (109) actually generates the conserved quantity (116) in the dipole approximation. Moreover, we will show that there exists a generalization of (116) to an arbitrary multipole order.

As it is well established, the equations of motion of extended test bodies in external gravitational and other classical fields are derived from the corresponding conservations laws, see [74-77] for example. In simple terms, the dynamical description of an extended body is achieved by assigning to a body a set (infinite, in general) of multipole moments. The latter are determined by certain integrals of the conserved currents (energymomentum tensor and other Noether currents) over the body. The multipole moments represent such characteristics of the body as its total mass, charge, momentum etc. By integrating the partial differential conservation laws, one obtains a system of ordinary differential equations of motion for the moments.

Here we apply the covariant multipole expansion of [76] to the current (106). The method is based on Synge's [78] notion of the "world function" $\sigma$, which introduces a covariant generalization of the finite distance between the spacetime points $x$ and $y$. Basic definitions and notation are summarized in Appendix C. By construction, this object $\sigma(x, y)$ depends on two arguments, and as a result the Synge formalism deals with arbitrary bitensor densities $\mathfrak{B}^{x_{1} y_{1}}=\mathfrak{B}^{x_{1} y_{1}}(x, y)$. The most important technical tool is represented by the lemma [76]:

$$
\begin{aligned}
\frac{D}{d s} \int_{\Sigma(s)} \mathfrak{B}^{x_{1} y_{1}} d \Sigma_{x_{1}}= & \int_{\Sigma(s)} \widetilde{\nabla}_{x_{1}} \mathfrak{B}^{x_{1} y_{1}} w^{x_{2}} d \Sigma_{x_{2}} \\
& +\int_{\Sigma(s)} v^{y_{2}} \widetilde{\nabla}_{y_{2}} \mathfrak{B}^{x_{1} y_{1}} d \Sigma_{x_{1}}
\end{aligned}
$$

We introduce the 4-velocity $v^{y_{1}}:=d x^{y_{1}} / d s$, with the proper time $s$, and denote $\frac{D}{d s}=v^{i} \widetilde{\nabla}_{i}$; the integrals are performed with an arbitrary bitensor density $\mathfrak{B}^{x_{1} y_{1}}(x, y)$ over an arbitrary spatial hypersurface $\Sigma$. See [76] for more details on the integrals and the construction of $w^{x}$. As in all our previous papers, we use a condensed notation suppressing the tensor indices so that $y_{n}$ denotes indices at the spacetime point $y$, etc.
Following the standard procedure, we define the multipole moments for the current (106):

$$
\begin{aligned}
j^{y_{1} \ldots y_{n}} & :=(-1)^{n} \int_{\Sigma(s)} \sigma^{y_{1}} \cdots \sigma^{y_{n}} \mathfrak{J}^{x^{\prime}} d \Sigma_{x^{\prime}} \\
i^{y_{1} \ldots y_{n} y_{0}} & :=(-1)^{n} \int_{\Sigma(s)} \sigma^{y_{1}} \cdots \sigma^{y_{n}} g^{y_{0}}{ }_{{ }_{0}} \mathfrak{J}^{x_{0}} w^{x^{\prime}} d \Sigma_{x^{\prime}} .
\end{aligned}
$$

Then, by applying the lemma (117), we integrate the balance equation (107). The result reads

$$
\begin{aligned}
& \frac{D}{d s} j^{a_{1} \ldots a_{n}}=-n v^{\left(a_{1}\right.} j^{\left.a_{2} \ldots a_{n}\right)}+n i^{\left(a_{1} \ldots a_{n}\right)} \\
& +\boldsymbol{Q}^{a_{1} \ldots a_{n} b_{c}{ }^{d}}\left(F \mathcal{L}_{\zeta} N_{d b}{ }^{c}\right)-\boldsymbol{\Xi}^{a_{1} \ldots a_{n}}\left(\mathcal{L}_{\zeta} F\right) .
\end{aligned}
$$

By boldface symbols we denote the following differentialalgebraic operators

$$
\begin{aligned}
\boldsymbol{Q}^{a_{1} \ldots a_{n} b{ }_{c}{ }^{d}} & =\sum_{k=0}^{\infty} \frac{1}{k !} q^{a_{1} \ldots a_{n+k} b}{ }_{c}{ }^{d} \widetilde{\nabla}_{a_{n+1}} \ldots \widetilde{\nabla}_{a_{n+k}}, \\
\boldsymbol{\Xi}^{a_{1} \ldots a_{n}} & =\sum_{k=0}^{\infty} \frac{1}{k !} \xi^{a_{1} \ldots a_{n+k}} \widetilde{\nabla}_{a_{n+1}} \ldots \widetilde{\nabla}_{a_{n+k}},
\end{aligned}
$$

which are defined in terms of the multipole moments $(\mathrm{C} 4)$ and (C5).

The current (106) has a nontrivial structure in that it is built from the Noether currents related to the diffeomorphism symmetry of the gravitational theory and of the auxiliary objects: vector fields $\zeta$ and the coupling function $F$. Taking this into account, we can use the lemma (117) once again to recast the moments into

$$
\begin{aligned}
j^{a_{1} \ldots a_{n}} & =\boldsymbol{P}^{a_{1} \ldots a_{n} b}\left(F \zeta_{b}\right)-\boldsymbol{H}^{a_{1} \ldots a_{n} b}{ }_{c}\left(F \bar{\nabla}_{b} \zeta^{c}\right),(123) \\
i^{a_{1} \ldots a_{n} a_{0}} & =\boldsymbol{K}^{a_{1} \ldots a_{n} b a_{0}}\left(F \zeta_{b}\right)-\boldsymbol{Q}^{a_{1} \ldots a_{n} b}{ }_{c}^{a_{0}}\left(F \bar{\nabla}_{b} \zeta^{c}\right) .
\end{aligned}
$$

Here we introduced new operators

$$
\begin{aligned}
\boldsymbol{P}^{a_{1} \ldots a_{n} b} & =\sum_{k=0}^{\infty} \frac{1}{k !} p^{a_{1} \ldots a_{n+k} b} \widetilde{\nabla}_{a_{n+1}} \ldots \widetilde{\nabla}_{a_{n+k}}, \\
\boldsymbol{H}^{a_{1} \ldots a_{n} b} & =\sum_{k=0}^{\infty} \frac{1}{k !} h^{a_{1} \ldots a_{n+k} b}{ }_{c} \widetilde{\nabla}_{a_{n+1}} \ldots \widetilde{\nabla}_{a_{n+k}}, \\
\boldsymbol{K}^{a_{1} \ldots a_{n} b c} & =\sum_{k=0}^{\infty} \frac{1}{k !} k^{a_{1} \ldots a_{n+k} b c} \widetilde{\nabla}_{a_{n+1}} \ldots \widetilde{\nabla}_{a_{n+k}},
\end{aligned}
$$

defined in terms of the multipole moments of the hypermomentum and the energy-momentum currents (C1)(C3).

Let us now analyze the moments equation (120). This is an infinite system of ordinary differential equations, which is common for the equations of motion of extended 
test bodies. However, one equation is a special one in this system. It corresponds to $n=0$ and reads explicitly

$$
\frac{d j}{d s}=Q_{c}^{b}{ }_{c}^{d}\left(F \mathcal{L}_{\zeta} N_{d b}^{c}\right)-\boldsymbol{\Xi}\left(\mathcal{L}_{\zeta} F\right)
$$

We immediately observe its similarity to (107). Moreover, the structure

$$
j=\boldsymbol{P}^{b}\left(F \zeta_{b}\right)-\boldsymbol{H}_{c}^{b}\left(F \bar{\nabla}_{b} \zeta^{c}\right)
$$

is obviously induced by the structure of the current (106).

After these general derivations, we are now in a position to specialize to the pole-dipole case. In the dipole approximation, only the first terms proportional to the multipole moments up to the dipole order - i.e. $p^{a}, p^{a b}$, $h^{a b}, k^{a b}, k^{a b c}, q^{a b c}, \xi$ and $\xi^{a}$ - need to be taken into account in (120)-(129). We then find for the equation (128) and for the moment (129):

$$
\begin{gathered}
\frac{d j}{d s}=q^{b}{ }_{c}^{d} F \mathcal{L}_{\zeta} N_{d b}{ }^{c}-\xi \mathcal{L}_{\zeta} F-\xi^{a} \widetilde{\nabla}_{a} \mathcal{L}_{\zeta} F \\
j=p^{a} F \zeta_{a}+p^{b a} \widetilde{\nabla}_{b}\left(F \zeta_{a}\right)-h^{b}{ }_{a} F \bar{\nabla}_{b} \zeta^{a}
\end{gathered}
$$

Making use of (110)-(112), we immediately see that these equations reproduce (115) and (116), respectively.

\section{CONCLUSIONS}

In this paper we have demonstrated that conserved currents can be naturally associated with spacetime diffeomorphisms (represented by vector fields on the spacetime manifold). Our findings extend previous results from Einstein's gravity with minimal coupling to the generalized metric-affine gravity theory with a possible nonminimal coupling.

Mathematically, an important role in this construction is played by the generalized Killing vector fields, which represent the symmetries on the metric-affine spacetime. The corresponding formalism is based on the fundamental geometrical notions of the transposed connection and of the Lie derivatives which we heavily used in our previous studies [27-29].

There are many physically interesting applications of the results obtained. Among them is the possibility of the rigorous computation of the total mass and angular momentum for the exact solutions in the gravitational theories with and without torsion and nonmetricity. Another application is to use the conserved quantity (116) to simplify the study of the dynamics of extended microstructured test bodies in the generalized gravitational field models. Both issues are important for gravitational experiments, including space missions, which aim for an advanced probe of the geometrical structure of spacetime.

The results of the present work should be used in the context of multipolar approximation schemes, which were recently worked out in $[68,79]$ for a very large class of gravitational theories, to further study the dynamics of test bodies. Of particular importance is the analysis of the detectability of post-Riemannian properties of spacetime, i.e. the torsion and the nonmetricity. It is worthwhile to mention that the recent literature on the Gravity Probe B experiment [80] encompasses the misleading claim that the GPB result may set limits on torsion [81, 82]. These erroneous statements were corrected in $[68,83-86]$, demonstrating that in minimally coupled gravitational theories the post-Riemannian geometry can only be detected with the help of microstructured matter.

One may notice that the conditions of the generalized Killing vectors (79) and (80) imposed on the spacetime geometry may be quite strong. This is similar to the situation for the manifolds that admit various types of collineations. However, even in absence of exact symmetries one can consider approximate spacetime symmetries along the lines of $[56,87]$. We leave the corresponding analysis for the future.

\section{ACKNOWLEDGEMENTS}

This work was supported by the Deutsche Forschungsgemeinschaft (DFG) through the grant SFB 1128/1 (D.P.), by Comisión Nacional de Investigación Científica y Tecnológica (Chile) through grant CONICYTPCHA/MagísterNacional/2014-22141453 (F.P-O.) and Vicerectoría de Investigación y Desarrollo UdeC (VRID) through grant 214.011.058-1.0 (G.R.).

\section{Appendix A: Notations and conventions}

In order to be consistent with our previous publications, we choose our main notations and conventions as those of [37]. Most importantly, we stick to the definitions of [37] for all the basic geometrical quantities such as the curvature, torsion, and nonmetricity, and we use the Latin alphabet to label the spacetime coordinate indices.

The spacetime is modeled as a four-dimensional smooth manifold, and its metric has the signature $(+,-,-,-)$. It should be noted though that our definition of the metrical energy-momentum tensor differs by a sign from the definition used in [88].

In this work we are widely using tensor densities; we denote the densities by the "Fraktur" font to distinguish them from the tensor objects.

Table I displays the list of symbols used in the current paper. 
TABLE I. Directory of symbols.

\begin{tabular}{|c|c|}
\hline Symbol & Explanation \\
\hline \multicolumn{2}{|c|}{ Geometrical quantities } \\
\hline$g_{a b}$ & Metric \\
\hline$\sqrt{-g}$ & Determinant of the metric \\
\hline$x^{a}, s$ & Coordinates, proper time \\
\hline$\zeta^{a}$ & Killing vector field \\
\hline$\Gamma_{a b}^{c}, \bar{\Gamma}_{a b}^{c}$ & Connection, transposed conn. \\
\hline$N_{a b}^{c}$ & Distortion \\
\hline$Q_{a b c}$ & Nonmetricity \\
\hline$T_{a b}{ }^{c}$ & Torsion \\
\hline$R_{a b c}{ }^{d}$ & Curvature \\
\hline$R_{a b}, R$ & Ricci tensor, scalar \\
\hline$\left(\sigma^{A}{ }_{B}\right)_{i}{ }^{j}$ & Generators coord. transf. \\
\hline$\delta_{b}^{a}$ & Kronecker symbol \\
\hline $\mathfrak{V}$ & Gravitational Lagrangian \\
\hline $\mathfrak{E}^{k i}{ }_{j}$ & Gravitational hypermomentum \\
\hline $\mathfrak{E}_{k}^{i}$ & Gener. grav. energy-momentum \\
\hline$\sigma, g^{y_{0}}{ }_{x_{0}}$ & World function, parallel propagator \\
\hline \multicolumn{2}{|l|}{ Matter quantities } \\
\hline$T^{a b}$ & Symmetric energy-momentum tensor \\
\hline$\psi^{A}$ & General matter field \\
\hline $\mathfrak{L}_{\text {mat }}$ & Matter Lagrangian \\
\hline $\mathfrak{J}^{a}$ & Generalized current \\
\hline $\mathfrak{K}^{i j}$ & Superpotential \\
\hline $\mathfrak{T}_{k}^{i}$ & Canonical energy-momentum \\
\hline $\mathfrak{S}_{j}^{i}{ }_{j}^{k}$ & Canonical hypermomentum \\
\hline$\tau_{i j}{ }^{k}$ & Spin density \\
\hline$t^{i j}$ & Metrical energy-momentum \\
\hline \multicolumn{2}{|l|}{ Auxiliary quantities } \\
\hline$\epsilon$ & Infinitesimal parameter \\
\hline$\varepsilon^{i}$ & Arbitrary vector field \\
\hline$\Phi^{J}$ & Multiplet of fields \\
\hline$I, \mathfrak{L}$ & General action, Lagrangian \\
\hline$F$ & Coupling function \\
\hline $\mathfrak{H}^{k l i}{ }_{j}, \mathfrak{H}^{k i}{ }_{j}, \mathfrak{M}^{k i j}$ & Gener. gravitational field momenta \\
\hline$p^{\cdots}, k^{\cdots}, h^{\cdots}, q^{\cdots}, \xi^{\cdots}$ & Test body integrated / generalized \\
\hline$L^{a b}, S^{a b}, \mathcal{P}^{a}, \mathcal{J}^{a b}, v^{a}$ & moments, velocity \\
\hline$\Omega^{\cdots}, w^{a}, \Phi^{\cdots}, \Psi^{\cdots}$ & Auxiliary variables \\
\hline \multicolumn{2}{|l|}{ Operators } \\
\hline $\mathcal{L}_{\zeta}$ & Lie derivative \\
\hline$\delta$ & Transformation under diffeomorph. \\
\hline$\delta_{(s)}$ & Substantial variation \\
\hline$\partial_{i}, \nabla_{i}$ & Partial, covariant derivative \\
\hline$\stackrel{*}{\nabla}_{i}$ & Modified cov. derivative \\
\hline$\widehat{\nabla}_{i}, \check{\nabla}_{i}$ & Cov. density derivative, Riemannian \\
\hline " & Riemannian quantity \\
\hline "— " & Transposed quantity \\
\hline$" \mathfrak{A} \cdots, \mathfrak{B} \cdots, \ldots "$ & Densities "Fraktur" \\
\hline $\boldsymbol{\Xi}^{\cdots}, \boldsymbol{H}^{\cdots}, \boldsymbol{K}^{\cdots}$ & Auxiliary diff. operators \\
\hline$P^{\cdots}, Q^{\cdots}$ & \\
\hline
\end{tabular}

\section{Appendix B: Diffeomorphism invariance}

The explicit structure of the functions $\Omega_{k}{ }^{i_{1} \cdots i_{n}}$, with $n=0,1,2,3$, is as follows

$$
\begin{aligned}
& \Omega_{k}=\partial_{i}\left(\frac{\partial \mathfrak{L}}{\partial \partial_{i} g_{m n}} \partial_{k} g_{m n}+\frac{\partial \mathfrak{L}}{\partial \partial_{i} \psi^{A}} \partial_{k} \psi^{A}-\delta_{k}^{i} \mathfrak{L}\right) \\
& +\frac{\delta \mathfrak{L}}{\delta g_{i j}} \partial_{k} g_{i j}+\frac{\delta \mathfrak{L}}{\delta \psi^{A}} \partial_{k} \psi^{A} \\
& +\frac{\partial \mathfrak{L}}{\partial \Gamma_{l n}{ }^{m}} \partial_{k} \Gamma_{l n}{ }^{m}+\frac{\partial \mathfrak{L}}{\partial \partial_{i} \Gamma_{l n}{ }^{m}} \partial_{k} \partial_{i} \Gamma_{l n}{ }^{m}, \\
& \Omega_{k}^{i}=2 \frac{\delta \mathfrak{L}}{\delta g_{i j}} g_{k j}+\frac{\delta \mathfrak{L}}{\delta \psi^{A}}\left(\sigma^{A}{ }_{B}\right)_{k}^{i} \psi^{B} \\
& +\frac{\partial \mathfrak{L}}{\partial \partial_{i} g_{m n}} \partial_{k} g_{m n}+\frac{\partial \mathfrak{L}}{\partial \partial_{i} \psi^{A}} \partial_{k} \psi^{A}-\delta_{k}^{i} \mathfrak{L} \\
& +\partial_{j}\left(2 \frac{\partial \mathfrak{L}}{\partial \partial_{j} g_{i n}} g_{n k}+\frac{\partial \mathfrak{L}}{\partial \partial_{j} \psi^{A}}\left(\sigma^{A}{ }_{B}\right)_{k}{ }^{i} \psi^{B}\right) \\
& +\frac{\partial \mathfrak{L}}{\partial \Gamma_{l i}^{j}} \Gamma_{l k}^{j}+\frac{\partial \mathfrak{L}}{\partial \Gamma_{i l}^{j}} \Gamma_{k l}^{j}-\frac{\partial \mathfrak{L}}{\partial \Gamma_{l j}^{k}} \Gamma_{l j}^{i} \\
& +\frac{\partial \mathfrak{L}}{\partial \partial_{i} \Gamma_{l n}{ }^{m}} \partial_{k} \Gamma_{l n}{ }^{m}+\frac{\partial \mathfrak{L}}{\partial \partial_{n} \Gamma_{i l}{ }^{m}} \partial_{n} \Gamma_{k l}{ }^{m} \\
& +\frac{\partial \mathfrak{L}}{\partial \partial_{n} \Gamma_{l i}{ }^{m}} \partial_{n} \Gamma_{l k}{ }^{m}-\frac{\partial \mathfrak{L}}{\partial \partial_{n} \Gamma_{l m}{ }^{k}} \partial_{n} \Gamma_{l m}{ }^{i}, \\
& \Omega_{k}^{i j}=\frac{4 \partial \mathfrak{L}}{\partial \partial_{(i} g_{j) n}} g_{k n}+\frac{2 \partial \mathfrak{L}}{\partial \partial_{(i} \psi^{A}}\left(\sigma^{A}{ }_{B}\right)_{k}^{j)} \psi^{B} \\
& +\frac{2 \partial \mathfrak{L}}{\partial \Gamma_{(i j)}{ }^{k}}+\frac{2 \partial \mathfrak{L}}{\partial \partial_{(i} \Gamma_{j) l}{ }^{m}} \Gamma_{k l}^{m} \\
& +\frac{2 \partial \mathfrak{L}}{\partial \partial_{(i} \Gamma_{|l| j)} m} \Gamma_{l k}^{m}-\frac{2 \partial \mathfrak{L}}{\partial \partial_{(i} \Gamma_{|l n|}{ }^{k}} \Gamma_{l n}{ }^{j)}, \\
& \Omega_{k}^{i j n}=\frac{\partial \mathfrak{L}}{\partial \partial_{n} \Gamma_{(i j)}^{k}}+\frac{\partial \mathfrak{L}}{\partial \partial_{i} \Gamma_{(j n)} k}+\frac{\partial \mathfrak{L}}{\partial \partial_{j} \Gamma_{(n i)}{ }^{k}} .
\end{aligned}
$$

\section{Appendix C: Multipole moments}

From the energy-momentum tensor density and the hypermomentum density, the integrated multipole mo- 
ments of arbitrary order, $n=0,1,2, \ldots$, are defined by

$$
\begin{aligned}
p^{y_{1} \ldots y_{n} y_{0}:=} & (-1)^{n} \int_{\Sigma(s)} \Phi^{y_{1} \ldots y_{n} y_{0}}{ }_{x_{0}} \mathfrak{T}^{x_{0} x_{1}} d \Sigma_{x_{1}},(\mathrm{C} 1) \\
k^{y_{2} \ldots y_{n+1} y_{0} y_{1}:=} & (-1)^{n} \int_{\Sigma(s)} \Psi^{y_{2} \ldots y_{n+1} y_{0} y_{1}}{ }_{x_{0} x_{1}} \times \\
& \times \mathfrak{T}^{x_{0} x_{1}} w^{x_{2}} d \Sigma_{x_{2}}, \\
h^{y_{2} \ldots y_{n+1} y_{0} y_{1}:=} & (-1)^{n} \int_{\Sigma(s)} \Psi^{y_{2} \ldots y_{n+1} y_{0} y_{1}}{ }_{x_{0} x_{1}} \times \\
& \times \mathfrak{S}^{x_{0} x_{1} x_{2}} d \Sigma_{x_{2}}, \\
q^{y_{3} \ldots y_{n+2} y_{0} y_{1} y_{2}:=} & (-1)^{n} \int_{\Sigma(s)} \Psi^{y_{3} \ldots y_{n+2} y_{0} y_{1}}{ }_{x_{0} x_{1}} g^{y_{2}} x_{x_{2}} \times \\
& \times \mathfrak{S}^{x_{0} x_{1} x_{2}} w^{x_{3}} d \Sigma_{x_{3}}, \\
\xi^{y_{1} \ldots y_{n}}:= & (-1)^{n} \int_{\Sigma(s)} \sigma^{y_{1}} \ldots \sigma^{y_{n}} \mathfrak{L}_{\mathfrak{m a t}} w^{x_{2}} d \Sigma_{x_{2}} .
\end{aligned}
$$

The integrals are taken over a cross-section $\Sigma(s)$ of the body's world tube. Here we introduced

$$
\begin{aligned}
\Phi^{y_{1} \ldots y_{n} y_{0}}{ }_{x_{0}} & :=\sigma^{y_{1}} \cdots \sigma^{y_{n}} g^{y_{0}}{ }_{x_{0}}, \\
\Psi^{y_{1} \ldots y_{n} y_{0} y^{\prime}}{ }_{x_{0} x^{\prime}}: & =\sigma^{y_{1}} \cdots \sigma^{y_{n}} g^{y_{0}}{ }_{x_{0}} g^{y^{\prime}}{ }_{x^{\prime}} .
\end{aligned}
$$

In the derivation of the equations of motion we made use of the bitensor formalism; see, e.g., [78, 89, 90] for introductions and references. In particular, the world function is defined as an integral $\sigma(x, y):= \pm \frac{1}{2}\left(\int_{x}^{y} d s\right)^{2}$ over the geodesic curve connecting the spacetime points $x$ and $y$, where the upper/lower sign is chosen for timelike/spacelike curves, respectively. Note that our curvature conventions differ from those in [78, 90]. Indices attached to the world function always denote covariant derivatives, at the given point, i.e. $\sigma_{y}:=\nabla_{y} \sigma$; hence, we do not make explicit use of the semicolon in the case of the world function. The parallel propagator by $g^{y}{ }_{x}(x, y)$ allows for the parallel transportation of objects along the unique geodesic that links the points $x$ and $y$. For example, given a vector $V^{x}$ at $x$, the corresponding vector at $y$ is obtained by means of the parallel transport along the geodesic curve as $V^{y}=g^{y}{ }_{x}(x, y) V^{x}$. For more details see, e.g., section 5 in [90]. A compact summary of useful formulas in the context of the bitensor formalism, as well as a review of the multipolar formalism employed here can also be found in [79].
[1] A. Komar. Covariant conservation laws in general relativity. Phys. Rev., 113:934, 1959.

[2] A. Komar. Asymptotic covariant conservation laws for gravitational radiation. Phys. Rev., 127:1411, 1962.

[3] V. Iyer and R. M. Wald. Some properties of the Noether charge and a proposal for dynamical black hole entropy. Phys. Rev. D, 50:846, 1994.

[4] I. M. Benn. Conservation laws in arbitrary spacetime. Ann. Inst. H. Poincaré A, 37:67, 1982.

[5] B. Julia and S. Silva. Currents and superpotentials in classical gauge-invariant theories: I. Local results with applications to perfect fluids and general relativity. Class. Quantum Grav., 15:2173, 1998.

[6] B. Julia and S. Silva. Currents and superpotentials in classical gauge-invariant theories: II. Global aspects and the example of affine gravity. Class. Quantum Grav., 17:4733, 2000.

[7] L. Fatibene, M. Ferraris, M. Francaviglia, and M. Raiteri. Remarks on conserved quantities and entropy of BTZ black hole solutions I. The general setting. Phys. Rev. D, 60:124012, 1999.

[8] M. Ferraris and M. Francaviglia. Covariant first-order Lagrangians, energy-density and superpotentials in general relativity. Gen. Rel. Grav., 22:965, 1990.

[9] M. Ferraris, M. Francaviglia, and M. Mottini. Conserved quantities of the gravitational field in tetrad notation. Rendiconti di Matematica (Rome), 14:457, 1994.

[10] M. Ferraris, M. Francaviglia, and M. Raiteri. Conserved quantities from the equations of motion: with applica- tions to natural and gauge natural theories of gravity. Class. Quantum Grav., 20:4043, 2003.

[11] J. Katz, D. Lynden-Bell, and J. Bicak. Gravitational energy in stationary spacetimes. Class. Quantum Grav., 23:7111, 2006.

[12] A. Borowiec, M. Ferraris, M. Francaviglia, and I. Volovich. Energy-momentum complex for nonlinear gravitational Lagrangians in the first order formalism. Gen. Rel. Grav., 26:637, 1994.

[13] P. T. Chruściel. On the relation between the Einstein and the Komar expressions for the energy of the gravitational field. Ann. Inst. H. Poincaré A, 42:267, 1985.

[14] A. N. Petrov and J. Katz. Conserved currents, superpotentials, and cosmological perturbations. Proc. Roy. Soc. Lond. A, 458:319, 2002.

[15] N. Deruelle and Y. Morisawa. Mass and angular momenta of Kerr anti-de Sitter spacetimes in EinsteinGauss-Bonnet theory. Class. Quantum Grav., 22:933, 2005.

[16] C.-C. Chang, J. M. Nester, and C.-M. Chen. Pseudotensors and quasilocal energy-momentum. Phys. Rev. Lett., 83:1897, 1999.

[17] C.-M. Chen and J. M. Nester. Quasilocal quantities for general relativity and other gravity theories. Class. Quantum Grav., 16:1279, 1999.

[18] J. M. Nester. General pseudotensors and quasilocal quantities. Class. Quantum Grav., 21:S261, 2004.

[19] G. Barnich and F. Brandt. Covariant theory of asymptotic symmetries, conservation laws and central charges. 
Nucl. Phys. B, 633:3, 2002.

[20] G. Giachetta and G. Sardanashvily. Stress-energymomentum of affine-metric gravity. Generalized Komar superpotential. Class. Quantum Grav., 13:L67, 1996.

[21] G. Sardanashvily. Stress-energy-momentum conservation law in gauge gravitation theory. Class. Quantum Grav., 14:1357, 1997.

[22] G. Giachetta, L. Mangiarotti, and G. Sardanashvily. Energy-momentum and gauge conservation laws. Gravitation and Cosmology, 5:92, 1999.

[23] E. Mielke. Affine generalization of the Komar complex of general relativity. Phys. Rev. D, 63:044018, 2001.

[24] R. D. Hecht, F. W. Hehl, E. W. Mielke, and Y. Ne'eman. Improved energy-momentum currents in metric-affine spacetimes. Phys. Lett. A, 172:13, 1992.

[25] R. Aros, M. Contreras, R. Olea, R. Troncoso, and J. Zanelli. Conserved charges for gravity with locally antide Sitter asymptotics. Phys. Rev. Lett., 84:1647, 2000.

[26] R. Aros, M. Contreras, R. Olea, R. Troncoso, and J. Zanelli. Conserved charges for even dimensional asymptotically AdS gravity theories. Phys. Rev. D, 62:044002, 2000.

[27] Yu. N. Obukhov and G. F. Rubilar. Invariant conserved currents in gravity theories with local Lorentz and diffeomorphism symmetry. Phys. Rev. D, 74:064002, 2006.

[28] Yu. N. Obukhov and G. F. Rubilar. Invariant conserved currents in gravity theories: Diffeomorphisms and local gauge symmetries. Phys. Rev. D, 76:124030, 2007.

[29] Yu. N. Obukhov and G. F. Rubilar. Invariant conserved currents for gravity. Physics Letters B, 660:240, 2008.

[30] A. N. Petrov and R. Lompay. Covariantized Noether identities and conservation laws for perturbations in metric theories of gravity. Gen. Rel. Grav., 45:545, 2013.

[31] R. Lompay and A. N. Petrov. Covariant differential identities and conservation laws in metric-torsion theories of gravitation. I. General consideration. J. Math. Phys., 54:062504, 2013.

[32] R. Lompay and A. N. Petrov. Covariant differential identities and conservation laws in metric-torsion theories of gravitation. II. Manifestly generally covariant theories. $J$. Math. Phys., 54:102504, 2013.

[33] J. A. Schouten. Ricci-Calculus. An introduction to tensor analysis and its geometric applications. Springer, Berlin, 2nd edition, 1954.

[34] J. L. Synge and A. Schild. Tensor calculus. Dover, New York, 1978.

[35] A. Lichnerowicz. Geometry of groups of transformations. Noordhoff International Publishing, Leyden, 1977.

[36] A. Trautman. On the Einstein-Cartan equations. IV. Bulletin de l'académie polonaise des sciences, sér. des sciences math., astr, et phys., 21:345, 1973.

[37] F. W. Hehl, J. D. McCrea, E. W. Mielke, and Y. Ne'eman. Metric-affine gauge theory of gravity: Field equations, Noether identities, world spinors, and breaking of dilation invariance. Phys. Rep., 258:1, 1995.

[38] D. W. Sciama. The analogy between charge and spin in general relativity. "Recent Developments in General Relativity", Festschrift for L. Infeld (Pergamon Press, Oxford; PWN, Warsaw), page 415, 1962.

[39] T. W. B. Kibble. Lorentz invariance and the gravitational field. J. Math. Phys., 2:212, 1961.

[40] F. Gronwald. Metric affine gauge theory of gravity. 1. Fundamental structure and field equations. Int. J. Mod. Phys. D, 6:263, 1997.
[41] Yu. N. Obukhov. Poincaré gauge gravity: selected topics. Int. J. Geom. Meth. Mod. Phys., 3:95, 2006.

[42] M. Blagojević. Gravitation and Gauge Symmetries. IOP Publishing, London, 2002.

[43] M. Blagojević and F. W. Hehl. Gauge Theories of Gravitation. A Reader with Commentaries. Imperial College Press, London, 2013.

[44] J. Dell, J. L. deLyra, and L. Smolin. Quantization of a gauge theory with independent metric and connection fields. Phys. Rev. D, 34:3012, 1986.

[45] C.-Y. Lee and Y. Ne'eman. Renormalization of gaugeaffine gravity. Phys. Lett. B, 242:59, 1990.

[46] C.-Y. Lee. Renormalization of quantum gravity with local $G L(4, R)$ symmetry. Class. Quantum Grav., 9:2001, 1992.

[47] C. Pagani and R. Percacci. Quantization and fixed points of non-integrable Weyl theory. Class. Quantum Grav., 31:115005, 2014.

[48] C. Pagani and R. Percacci. Quantum gravity with torsion and non-metricity. Class. Quantum Grav., 32:195019, 2015.

[49] K. Yano. The theory of Lie derivatives and its applications. North-Holland, Amsterdam, 1955.

[50] Y. Kosmann. Dérivées de Lie des spineurs. Comptes Rendus Acad. Sci. Paris A, 262:289, 1966.

[51] Y. Kosmann. Dérivées de Lie des spineurs. Applications. Comptes Rendus Acad. Sci. Paris A, 262:394, 1966.

[52] G. H. Katzin, J. Levine, and W. R. Davis. Curvature collineations: a fundamental symmetry property of the spacetimes of general relativity defined by the vanishing Lie derivative of the Riemann curvature tensor. J. Math. Phys., 10:617, 1969.

[53] G. S. Hall. Symmetries and curvature structure in general relativity. World Scientific, Singapore, 2004.

[54] C. D. Collinson. Conservation laws in general relativity based upon the existence of preferred collineations. Gen. Rel. Grav., 1:137, 1970.

[55] S. Hojman, L. Nuñez, A. Patiño, and H. Rago. Symmetries and conserved quantities in geodesic motion. $J$. Math. Phys., 27:281, 1986.

[56] A. I. Harte. Approximate spacetime symmetries and conservation laws. Class. Quantum Grav., 25:205008, 2008.

[57] F. W. Hehl, G. D. Kerlick, and P. von der Heyde. On hypermomentum in general relativity. I. The notion of hypermomentum. Zeits. Naturforsch., 31a:111, 1976.

[58] F. W. Hehl, G. D. Kerlick, and P. von der Heyde. On hypermomentum in general relativity. II. The geometry of spacetime. Zeits. Naturforsch., 31a:524, 1976.

[59] F. W. Hehl, G. D. Kerlick, and P. von der Heyde. On hypermomentum in general relativity. III. Coupling hypermomentum to geometry. Zeits. Naturforsch., 31a:823, 1976.

[60] F. W. Hehl and G. D. Kerlick. Metric-affine variational principles in general relativity. I. Riemannian spacetime. Gen. Rel. Grav., 9:691, 1978.

[61] F. W. Hehl and G. D. Kerlick. Metric-affine variational principles in general relativity. II. Relaxation of the Riemannian constraint. Gen. Rel. Grav., 13:1037, 1981.

[62] V. N. Ponomariev and Yu. N. Obukhov. The generalized Einstein-Maxwell theory of gravitation. Gen. Rel. Grav., 14:309, 1982.

[63] D. Vassiliev. Quadratic metric-affine gravity. Ann. Phys. (Berlin), 14:231, 2005. 
[64] T. P. Sotiriou and S. Liberati. Metric-affine $f(R)$ theories of gravity. Ann. Phys. (Amsterdam), 322:935, 2007.

[65] T. P. Sotiriou and V. Faraoni. $f(R)$ theories of gravity. Rev. Mod. Phys., 82:451, 2010.

[66] V. Vitagliano, T. P. Sotiriou, and S. Liberati. The dynamics of metric-affine gravity. Ann. Phys. (Amsterdam), 326:1259, 2011.

[67] Y. Ne'eman and F. W. Hehl. Test matter in a spacetime. Class. Quantum Grav., 14:A251, 1997.

[68] D. Puetzfeld and Yu. N. Obukhov. Equations of motion in metric-affine gravity: a covariant unified framework. Phys. Rev. D, 90:084034, 2014.

[69] H. P. Künzle. Canonical dynamics of spinning particles in gravitational and electromagnetic fields. J. Math. Phys., 13:739, 1972.

[70] S. Hojman. Electromagnetic and gravitational interactions of a relativistic top. PhD. thesis, Princeton University, 1975.

[71] J. Ehlers and E. Rudolph. Dynamics of extended bodies in general relativity: Center-of-mass description and quasirigidity. Gen. Rel. Grav., 8:197, 1977.

[72] Y. N. Obukhov and D. Puetzfeld. Dynamics of test bodies with spin in de Sitter spacetime. Phys. Rev. D, 83:044024, 2011.

[73] E. Hackmann, C. Lämmerzahl, Yu. N. Obukhov, D. Puetzfeld, and I. Schaffer. Motion of spinning test bodies in Kerr spacetime. Phys. Rev. D, 90:064035, 2014.

[74] M. Mathisson. Neue Mechanik materieller Systeme. Acta Phys. Pol., 6:163, 1937.

[75] A. Papapetrou. Spinning test-particles in General Relativity. I. Proc. Roy. Soc. Lond. A, 209:248, 1951.

[76] W. G. Dixon. A covariant multipole formalism for extended test bodies in General Relativity. Nuovo Cimento, 34:317, 1964.

[77] W. G. Dixon. Description of extended bodies by multipole moments in special relativity. J. Math. Phys., 8:1591, 1967.

[78] J. L. Synge. Relativity: The general theory. NorthHolland, Amsterdam, 1960.
[79] Yu. N. Obukhov and D. Puetzfeld. Multipolar test body equations of motion in generalized gravity theories. "Equations of Motion in Relativistic Gravity", D. Puetzfeld et. al. (eds.), Fundamental theories of Physics, Springer, 179:67, 2015.

[80] C.W. Will. Finally, results from Gravity Probe B. Physics, 4:43, 2011.

[81] K. Hayashi and T. Shirafuji. Frame-dragging precession of orbiting gyroscopes in new general relativity and possible violation of equivalence principle. Prog. Theor. Phys., 84:1074, 1990.

[82] Y. Mao, M. Tegmark, A. Guth, and S. Cabi. Constraining torsion with Gravity Probe B. Phys. Rev. D, $76: 104029,2007$.

[83] É.É. Flanagan and E. Rosenthal. Can Gravity Probe B usefully constrain torsion gravity theories? Phys. Rev. D, 75:124016, 2007.

[84] D. Puetzfeld and Yu. N. Obukhov. Propagation equations for deformable test bodies with microstructure in extended theories of gravity. Phys. Rev. D, 76:084025, 2007.

[85] D. Puetzfeld and Yu. N. Obukhov. Probing nonRiemannian spacetime geometry. Phys. Lett. A, 372:6711, 2008.

[86] F. W. Hehl, Yu. N. Obukhov, and D. Puetzfeld. On Poincaré gauge theory of gravity, its equations of motion, and Gravity Probe B. Phys. Lett. A, 377:1775, 2013.

[87] R. A. Matzner. Almost symmetric spaces and gravitational radiation. J. Math. Phys., 9:1657, 1968.

[88] D. Puetzfeld and Yu. N. Obukhov. Covariant equations of motion for test bodies in gravitational theories with general nonminimal coupling. Phys. Rev. D, 87:044045, 2013.

[89] B. S. DeWitt and R. W. Brehme. Radiation damping in a gravitational field. Ann. Phys (N.Y.), 9:220, 1960.

[90] E. Poisson, A. Pound, and I. Vega. The motion of point particles in curved spacetime. Living Reviews in Relativity, 14(7), 2011. 